\title{
6. Studien über die Anomalien im Verhalten der Dielektrika ${ }^{1}$ ); von Egon Ritter v. Schweidler.
}

\section{Einleitung.}

Vom Standpunkt der allgemeinen Theorie der elektrischen Erscheinungen ist ein homogenes und isotropes Dielektrikum durch zwei Materialkonstanten ausreichend zu charakterisieren: durch seine Dielektrizitätskonstante und durch sein spezifisches Leitvermögen.

Viele Dielektrika zeigen nun Anomalien ihres Verhaltens, die sich nicht ohne weiteres in den Rahmen der allgemeinen Theorie fassen lassen, ähnlich wie dies auf dem Gebiet des Magnetismus bei den ferromagnetischen Substanzen der Fall ist. Da sich ohne spezielle Hypothesen nachweisen läBt, daß die verschiedenen Arten der Anomalien in gegenseitigem Zusammenhange stehen, sind sie hier auch im Zusammenhange behandelt.

Die hier gegebene Darstellung zerfällt in drei Hauptteile.

Im ersten Teile werden die Hauptformen des anomalen Verhaltens der Dielekrika auf Grund der bisher vorliegenden experimentellen Ergebnisse ohne Anwendung einer speziellen Hypothese oder Theorie zusammengestellt; als solche Hauptformen werden unterschieden:

1. die Rückstandsbildung, das ist das Auftreten nach bestimmten Gesetzen zeitlich variabler Ströme in Dielektrikas unter der Einwirkung eines konstanten oder sehr langsam veränderlichen elektrischen Feldes;

2. die Energieverluste (Umwandlung elektrischer Energie in Wärme) in Dielektrikas unter dem EinfluB eines Wechseloder Drehfeldes;

1) Aus den Sitzungsber. der kaiserl. Akad. der Wissensch. in Wien. Mathem.-naturw. Kl. 116. p. 1055. 1907. 
3. die ponderomotorischen Kräfte, die ein Dielektrikum im elektrischen Drehfeld erfährt;

4. die scheinbare Abhängigkeit der Kapazität eines Kon. densators und somit auch der Dielektrizitätshonstante seines Dielektrikums von der Ladungsdauer bei konstanter Spannung, bzw. Periodendauer bei Wechselspannung.

Der Ausdruck "Hysteresis", der für die unter 2 , und 3. genannten Erscheinungen häufig gebraucht wird, ist hier vorläufig vermieden, da er bereits eine nicht allgemein angenommene Voraussetzung über die Natur dieser Phänomene zur Grundlage hat.

Der zweite Teil behandelt die Theorie der anomalen Er. scheinungen. Es wird zunächst der oben erwähnte gegenseitige Zusammenhang der vier Hauptformen abgeleitet; hierauf wird die Unvereinbarkeit der Anomalien mit den einfachen Annahmen der allgemeinen Theorie nachgewiesen; es folgt eine Diskussion der verschiedenen Möglichkeiten, diese Anomalien theoretisch zu behandeln durch Zurückführung auf Anomalien der Struktur des Mediums (Inhomogenität, Maxwellsche Theorie der geschichteten Dielektrika) oder der Leitungsvorgänge (Ionenleitung) oder endlich des dielektrischen Verhaltens (Nachwirkung, Hysteresis, Viskosität).

Der Versuch einer Modifikation der einzigen bisher vorliegenden präzisen Formulierung einer solchen Theorie (der Pellatschen Theorie) bildet zusammen mit dem Nachweis, dab aus den Gesetzen der Rückstandsbildung die übrigen Formen des anomalen Verhaltens der Dielektrika quantitativ darstellbar sind, den wesentlichsten Bestandteil dieser Studien.

Der dritte Teil enthält die Resultate experimenteller Untersuchungen des Verfassers. Da nach seiner Ansicht die bisher vorliegenden, im ersten Teile besprochenen Ergebnisse ausreichen, um die im zweiten Teile dargelegten theoretischen Folgerungen zu stützen, bieten sie nichts prinzipiell Neues, sondern dienen bloB zur Erläuterung, Bestätigung und Ergänzung jener Ergebnisse und zur Aufstellung eines Schemas für die Charakterisierung eines Dielektrikums durch Angabe seiner Materialkonstanten.

Eine Zusammenfassung der Ergebnisse schlieBt die vorliegenden Studien ab. 
Der Anhang enthält ein Verzeichnis der einschlägigen Literatur, auf das die Zitate innerhalb des Textes bezogen sind.

\section{Erster Teil.}

Die Hauptformen des anomalen Verhaltens der Dielektrika.

1. Die Rückstandsbildung.

Werden die Belegungen eines Kondensators mit den Polen einer Stromquelle konstanter elektromotorischer Kraft verbunden, so tritt in den Zuleitungen ein Strom auf, dessen Intensität mit der Zeit abnimmt. Bei einem idealen nichtleitenden Dielektrikum gilt nach der allgemeinen Theorie für den ,normalen Ladungs-

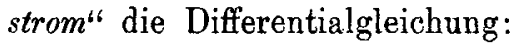

$$
S \frac{d^{2} i}{d t^{2}}-W-\frac{d i}{d t}+{ }_{C}^{1} i=0,
$$

wobei $C$ die Kapazität des Kondensators, $S$ den Selbstinduktionskoeffizienten und $W$ den Widerstand des äuBeren Leitungskreises darstellt. Je nach dem Verhältnis der numerischen Werte dieser drei Konstanten erfolgt die Ladung des Kondensators gedämpft oszillatorisch oder aperiodisch gedämpft. Bei nicht sehr groBem Widerstande des äuBeren Leitungskreises sinkt die Stromstärke in jedem der beiden Fälle sehr rasch $a b$, so daB in den praktisch realisierbaren Fällen der normale Ladungsstrom nach Zeiten von der Größenordnung eines kleinen Bruchteiles einer Sekunde gleich Null gesetzt werden kann.

Falls das Dielektrikum nicht vollkommen isolierend ist, so ist dem normalen Ladungsstrom ein "normaler Leitungs-

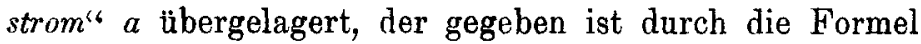

$$
a=\frac{4 \pi \lambda}{K} \cdot C E,
$$

wobei $\lambda$ das spezifische Leitvermögen, $K$ die Dielektrizitätskonstante des Mediums, $C$ die Kapazität des Kondensators und $E$ die elektromotorische Kraft der Stromquelle bezeichnet (alle Größen sind in absoluten elektrostatischen Einheiten gemessen gedacht).

Tatsächlich nun beobachtet man bei vielen Dielektrikas, daB dem normalen Ladungsstrom $i_{1}$ und dem normalen Leitungs- 
strom a noch ein ,,anomaler Ladungstrom" $y_{1}$ übergelagert ist, so daB der gesamte Strom darstellbar ist durch:

$$
J_{1}=i_{1}+y_{1}+a \text {. }
$$

Dabei ist $y_{1}$ eine Funktion der Zeit, die asymptotisch auf Null absinkt, aber viel langsamer als der normale Ladungsstrom $i_{1}$.

Werden die Belegungen des Kondensators, nachdem sie durch ein Zeitintervall $\delta$ auf konstanter Potentialdifferenz gehalten wurden, miteinander leitend verbunden, so sind bezüglich der Gesetze des Entladungsstromes zwei Typen zu unterscheiden:

a) Der Entladungsstrom $J_{2}$ entspricht dem normalen Entladungsstrom $i_{2}$, der durch eine ganze analoge Differentialgleichung wie der normale Ladungsstrom $i_{1}$ bestimmt ist;

b) analog wie bei der Ladung ist ein ,anomaler Entladungsstrom" $y_{2}$ übergelagert und es ist $y_{2}$ wieder eine mit wachsender Zeit auf Null absinkende Funktion dieser. Dabei gilt noch die Beziehung zwischen $y_{1}$ und $y_{2}$ :

$$
\int_{0}^{\delta} y_{1} d t=-\int_{0}^{\infty} y_{2} d t,
$$

d. h. die gesamte Elektrizitätsmenge, die infolge des anomalen Entladungsstromes einen Querschnitt der Leitung passiert, ist entgegengesetzt gleich derjenigen, die während der Ladungsdauer $\delta$ durch den anomalen Ladungsstrom transportiert wurde. Während im Falle a), der besonders bei verschiedenen schlecht leitenden Flüssigkeiten realisiert ist (Koll er $, I V, 3 ; \mathrm{Sch}$ w eid ler, IV, 7, 8, 10; Gädeke, IV, 9), der Ladungsprozeb so verläuft, als ob das Leitvermögen des Mediums durch den Stromdurchgang zeitliche Änderungen erfahren würde, ist im Falle b) der LadungsprozeB ein reversibler; das Medium verhält sich, als ob die dem Zeitintegral des anomalen Ladungsstromes $\int_{0}^{\delta} y_{1} d t$ entsprechende Elektrizitätsmenge absorbiert wäre, um bei der Entiadung allmählich wieder frei zu werden.

Diesen reversiblen ProzeB bezeichnet man gewöhnlich als Bildung bzw. Freiwerden des "Rückstandes" und das Integral $\int_{0}^{\delta} y_{1} d t$ als die in der Zeit $\delta$ gebildete "Rüchstandsladung". 
Prinzipiell weniger einfach sind die Verhältnisse, wenn die Belegungen des Kondensators nicht auf konstanten Potentialen gehalten werden, sondern eine von ihnen isoliert ist; doch ist gerade diese Form der Rückstandserscheinungen die historisch primäre und bei dieser wurde man auf die oben erwähnte Bezeichnungsweise geführt. Wird ein Kondensator geladen und dann die eine der beiden Belegungen isoliert, so nimmt die Potentialdifferenz $V$ der Belegungen und damit die durch das Produkt $C V$ gegebene sogenannte "disponible Ladung" ab. Diese Abnahme erfolgt rascher, als es dem stationären Leitungsstrom $a$ entsprechen würde. Wird umgekehrt der geladene Kondensator durch vorïbergehende leitende Verbindung der Belegungen entladen und hierauf die eine Belegung isoliert, so tritt allmäblich eine neuerliche Ladung vom gleichen. Vorzeichen wie die ursprüngliche auf, wächst bis zu einem Maximum an, um dann wieder (infolge der Leitung des Dielektrikums) asymptotisch auf Null zu sinken.

Bezüglicb der Gesetze, die für den zeitlichen Verlauf der Rückstandserscheinungen sowie für ihre Abhängigkeit von anderen Nebenbedingungen gelteu, haben die experimentellen Untersuchungen zu folgenden Resultaten geführt:

Der anomale Ladungsstrom oder rückstandsbildende Strom $y_{1}$ ist an einem gegebenen Kondensator bei gegebener elektromotorischer Kraft als Funktion der Zeit darstellbar durch:

Dabei ist $n<1$.

$$
y_{1}=B \cdot t^{-n} \text {. }
$$

Diese Formel kann nur als Annäherung betrachtet werden. Zunächst erhält man für $t=0, y_{1}=\infty$, wobei allerdings

$$
\int_{0}^{\delta} y_{1} d t=\frac{T 3 \cdot t^{1-n}}{1-n}
$$

für endliches $\delta$ endlich bleibt. Ferner wird für $t=\infty$,

$$
\int_{0}^{\infty} y_{1} d t=\infty .
$$

was ebenfalls aus später zu besprechenden Gründen unwahrscheinlich ist. Der Verlauf des Stromes innerhalb des ersten unendlich kleinen Zeitelementes sowie nach unendlich langer 
Zeit läBt sich natürlich nicht empirisch bestimmen. Innerhalb der der Beobachtung zugänglichen Zeitintervalle hat sich aber obige Formel gut bestätigt gezeigt (Kohlrausch, I, 1; Hopkinson, I, 9, 10, 11; Giese, I, 15; J. Curie, I, 21 u. a.) Versuche, welche über weit längere Zeiträume als in den zitierten Arbeiten die Gültigkeit dieser Formel prüfen, sind im dritten Teile angeführt.

Wird derselbe Kondensator unter der Einwirkung verschiedener elektromotorischer Kräfte untersucht, so ergibt sich. daB der Rückstandsstrom $y_{1}$ der elektromotorischen Kraft $E$ proportional ist bei unveränderter Form des zeitlichen Verlaufes. Also

$$
y_{1}=B \cdot t^{-n}=b \cdot E \cdot t^{-n}
$$

(Kohlrausch, I, 1; J. Curie, I, 21).

Wird ein bestimmtes Dielektrikum in verschiedenen Schichtdicken untersucht, so ist nach Hopkinson (I, 9, 10, 11) der Rückstandsstrom $y_{1}$ der Dicke umgekehrt proportional, wieder bei unveränderter Form des zeitlichen Verlaufes. Da Proportionalität des Stromes mit dem Querschnitt (Flächengröße der Belegung) selbstrerständlich ist, kann man auch den Strom proportional der Kapazität des Kondensators setzen und man erhält die Formel:

$$
y_{1}=b \cdot E \cdot t^{-n}=\beta \cdot C \cdot E \cdot t^{-n}
$$

wo jetzt $\beta$ und $n$ Konstanten des Mediums als solchen sind.

Bezüglich des Entladungsstromes $y_{2}$ gelten folgende Gesetze: Nach sehr langer Ladungsdauer des Kondensators ist: der Entladungsstrom dem Ladungsstrom entgegengesetzt gleich:

$$
y_{2}=-y_{1}
$$

bei kürzerer Ladungsdauer $\delta$ ist $y_{2}<y_{1}$, zeigt rascheren zeitlichen Abfall, und zwar darstellbar durch die Formeln:

$$
\begin{aligned}
& y_{1}=f(t)=B \cdot t^{-n}, \\
& y_{2}=-f(t)+f(t+\delta),
\end{aligned}
$$

wobei $t$ die rom Beginn der Ladung (bei $y_{1}$ ) bzw. Entladung (bei $y_{2}$ ) gerechnete Zeit, $\delta$ die Ladungsdauer bezeichnet.

Dieses Ergebnis kann so interpretiert werden: Der beobachtete Entladungsstrom $y_{2}$ entsteht aus der Superposition des dem Ladungsstrom $y_{1}=f(t)$ entgegengesetzt gleichen Stromes 
- $f(t)$ und eines Stromes $f(t+\delta)$, der einfach als ungestörte Fortsetzung des Ladungsstromes auch nach der Entladung aufgefaBt werden kann.

Dieses sogenannte Superpositionsprinzip wurde experimentell gefunden von Hopkinson $(\mathrm{I}, 9,10,11)$ und J.Curie (I, 21).

Die oben erwähnte Formel

$$
\int_{0}^{\infty} y_{2} d t=-\int_{0}^{\delta} y_{1} d t
$$

ist eine Konsequenz desselben.

Das Superpositionsprinzip kann noch erweitert werden: Bei mehrmaligen sprungweisen Änderungen der Spannungsdifferenz der Belegungen superponieren sich die jeder Änderung entsprechenden Ströme, als ob sie unabhängig voneinander wären. Wenn also zu den Zeiten

$$
t=\delta_{1}, \delta_{2}, \delta_{3} \ldots \delta_{k}
$$

Sprünge in der Potentialdifferenz um die (positiven oder negativen) Beträge:

$$
E_{1}, E_{2}, E_{3} \ldots E_{k}
$$

eintreten, so ist der resultierende Strom:

$$
y=E_{1} f^{\prime}\left(t-\delta_{1}\right)+E_{2}^{\prime} f^{\prime}\left(t-\delta_{2}\right)+\ldots E_{k} f\left(t-\delta_{k}\right) .
$$

Eine Verallgemeinerung dieses empirisch gefundenen und bestätigten Superpositionsprinzipes führt zur Darstellung des Stromes bei beliebig variierender Spannungsdifferenz

durch die Formel

$$
E=\varphi(t)
$$

$$
y_{t}=\int_{-\infty}^{t} \frac{d \varphi(\vartheta)}{d \dot{\vartheta}} \cdot f(t-\vartheta) d \vartheta .
$$

Darin ist $f(t)$ die Funktion, die den Verlauf nach einer einzigen sprungweisen Änderung im absoluten Betrage 1 darstellt, also nach dem oben Mitgeteilten:

$$
\text { annähernd } f(t)=\beta . C \cdot t^{-n} \text {. }
$$

Wie alle Materialkonstanten sind auch die den Verlauf der Rückstandsbildung bestimmenden Konstanten $\beta$ und $n$ als 
Funktionen der Temperatur zu betrachten. Die Angaben verschiedener Beobachter (Bedell und Kinsley, I, 26; Hopkinson und Wilson, I, 34; Naccari, IV, 6 und I, 37; Schweidler, I, 46) über den EinfluB der Temperatur zeigen Widersprüche, die aber vielleicht nur scheinbare sind. Gewöhnlich wird angegeben, daß der Rückstand mit steigender Temperatur abnehme; andere Beobachter geben an, dab mit steigender Temperatur die Konstante $\beta$ zunehme, während der Exponent $n$ nicht wesentlich von der Temperatur beeinflußt werde. Aus dieser letzteren Angabe würde folgen, daß die in gegebener Zeit $\delta$ gebildete Rückstandsladung

mit der Temperatur wachse.

$$
R=\beta \cdot C \cdot E \cdot \frac{t^{1-n}}{1-n}
$$

Wird aber nach längerer Ladungsdauer der Kondensator momentan entladen, hierauf die eine Belegung isoliert und nun mittels einer elektrometrischen Methode die bis $\mathrm{zu}$ einem Maximum ansteigende, dann wieder abnehmende Rückstandsladung messend verfolgt, so kann bei höherer Temperatur das beobachtete Maximum erniedrigt sein, wenn nämlich das Leitvermögen des Dielektrikums mit der Temperatur rascher ansteigt als die Größe $\beta$. Einige Versuche über diese Frage sind im dritten Teile besprochen.

Erwähnenswert sind noch zwei Beobachtungen über die Beeinflussung der Rückstandsbildung durch mechanische und elektrische Zustandsänderungen.

Nach Hopkinson $(I, 10)$ werden die anomalen Ladungsund Entladungsströme bei einer Leydnerflasche verstärkt durch gleichzeitige mechanische Erschütterungen.

Nach v. Hoor (VI, 2) kann die Rückstandsbildung eines Kondensators verringert werden durch oft wiederholtes „Formieren", d. i. abwechselndes Laden und Entladen.

\section{Energieumwandlung im Wechsel- oder Drehfelde.}

Kondensatoren mit flüssigem oder festem Dielektrikum zeigen häufig eine Erwärmung, wenn ihre Belegungen durch Verbindung mit einer Wechselstromquelle alternierend geladen werden (Siemens, II, 1; Naccari und Bellati, II, 2; Borgmann, II, 3; Steinmetz, II, 6; Janet, II, 8; Kleiner, 
II, 11; Fritz, II, 14; Düggelin, II, 21; Benischke, II, 24; Eisler, II, 25; Rosa und Smith, II, 35; Mercanton, II, 41; Moscicki und Altenberg, II, 49). Dasselbe findet statt, wenn ein Dielektrikum sich in einem elektrischen Drehfelde befindet (Guye und Denso, II, 50).

Bezüglich der verschiedenen Stärke dieser Wärmeproduktion in verschiedenen Substanzen sind besonders die Untersuchungen von Kleiner (II, 11), Fritz (II, 14) und Düggelin (II, 21) zu nennen. Kautschuk, Ebonit, Glas, Siegellack und Guttapercha zeigen diese Erscheinung in besonderer Intensität, Glimmer, Paraffin und viele isolierende Flüssigkeiten in schwachem bis unmerklichem Grade.

Eine Reihe von Untersuchungen betrifft die Abhängigkeit von der Effektivspannung des Wechselfeldes; die meisten Beobachter finden Proportionalität zwischen der pro Zeiteinheit entwickelten Wärme $W$ und dem Quadrat der Effektivspannung $E$ (Borgmann, II, 3; Steinmetz, II, 6; Hess, II, 23; Benischke, II, 24; Eisler, II, 25; Houllerigue, II, 29); doch finden andere Beobachter (Mercanton, II, 41; Moscicki und Altenberg, II, 49) auch Abweichungen von diesem Gesetze.

Weitere Resultate betreffen die Abhängigkeit von der Periodenzahl $n$ des angewandten Wechselstromes. Im allgemeinen steigt die Wärmemenge $W$ mit der Periodenzahl an (Hess, II, 23; Eisler, II, 25; Beaulard, II, 36 und 37; Moscicki und Altenberg, II, 49; Guye und Denso, II, 50). Wird die entwickelte Wärmemenge auf die Dauer der Periode des Wechselstromes $\tau$ bezogen statt auf die Zeiteinheit, so ergibt sich, daB die Funktion $\tau W=f(n)$ bei einem gewissen Werte von $n$ ein Maximum besitzt (Mercanton, II, 41; Rosa und Smith, II, 35).

Analog wie beim Rückstand kann bisweilen die Wärmeproduktion im Dielektrikum durch wiederholte Beanspruchung (,Formieren“) herabgedrückt werden (Kleiner, II, 20; v. Hoor, $\nabla I, 2)$.

\section{Ponderomotorische Kräfte im elektrischen Drehfelde.}

Diese Erscheinungen treten auf bei relativer Rotation des Dielektrikums zu einem elektrischen Felde, also entweder wenn 
ein ruhendes Dielektrikum sich in einem elektrischen Drehfelde befindet oder wenn ein Dielektrikum in einem ruhenden Felde rotiert. Es zeigt sich, daB in diesen Fällen Drehungsmomente auf das Dielektrikum ausgeübt werden, auch wenn es die Form eines Rotationskörpers mit zur Feldrichtung senkrechter Achse hat, also aus Symmetriegründen bei relativer Ruhe keine Drehungsmomente entstehen können (Arno, II, 7, 15-19, 27; Quincke, III, 2; Borel, II, 12; Threlfall, II, 30, 31; Schaufelberger, II, 32, 34; Heydweiller, III, 6; Graetz, III, 7; v. Lang, II, 52).

Ist das Dielektrikum von Luft umgeben, so entsteht ein Drehungsmoment, das es im Sinne der Rotation des Feldes zu drehen sucht: also Mitnehmen des ursprünglich ruhenden Dielektrikums im rotierenden Felde oder Hemmung des rotieren. den Dielektrikums im ruhenden Felde.

Ist aber der Körper in eine sehr schlecht leitende Flüssigkeit eingetaucht, so kann sich das Vorzeichen des Drehungsmomentes umkehren: im rubenden Felde wird eine vorhandene Rotation des Körpers beschleunigt (Quincke, III, 2), im Drehfelde eine der Feldrotation entgegengesetzte Rotation des Körpers hervorgerufen (v. Lang, II, 52).

Eine quantitative Bestimmung des Drehmomentes kann erfolgen im Drehfelde aus der Torsion einer elastischen Suspension, die dem elektrischen Drehmomente das Gleichgewicht hält, im ruhenden Felde aus der Dämpfung von Schwingungen (Arno, Threlfall, Schaufelberger). Für die Abhängigkeit des Drehmomentes $D$ von der elektrischen Feldintensität $F$ findet Schaufelberger die Beziehung:

$$
D=k \cdot F^{2}
$$

A rno und Threlfall dagegen:

$$
D=K \cdot F^{n}
$$

wobei $n$ in verschiedenen Fällen verschieden $(1,5$ bis 1,96$)$, aber immer kleiner als 2 war.

Bei konstanter Feldintensität nimmt das Drehungsmoment mit wachsender Rotationsgeschwindigkeit zu (Arno, II, 27). 


\section{Abhängigkeit der Dielektrizitätskonstante von Ladungs- dauer bzw. Periodenzahl.}

Sowohl wenn die Kapazität eines Kondensators bei kur\%dauernder Verbindung mit einer Stromquelle konstanter Spannung bestimmt wird als auch bei Verwendung einer Wechselstrommethode ergaben viele Versuche eine Abhängigkeit des unmittelbar gefundenen Wertes für die. Kapazität und somit auch des Wertes der Dielektrizitätskonstante von der Ladungsdauer bzw. von der Periodenzahl (Frequenz) des Wechselstromes (vgl. Literaturverzeichnis, Abteilung V).

Mit einer einzigen Ausnahme (Lecher, V, 6) fanden alle Beobachter, daB die Dielektrizitätskonstante um so kleiner wird, je kürzer die Ladungsdauer, bzw. je größer die Periodenzahl ist. Bisweilen sind die Unterschiede sebr bedeutend; z. B. findet J. J. Thomson $(\mathrm{V}, 5)$

für Glas, bei Frequenz $n=$ ca. $100^{1 / \mathrm{sec}:} K=9$ bis 11 ,

$$
\text { " } \quad n=25 \cdot 10^{6}: K=2,7 \text {; }
$$

analog Northrup $(\nabla, 17)$

$$
\text { für Glas, bei } \begin{array}{rlrl}
n & =100 \quad: \quad K=6,25, \\
\eta & n=10^{6} \text { bis } 10^{7}: & K=5,86 ;
\end{array}
$$

and Beaulard $(V, 15)$

für $Q l a s$, bei Ladungsdauer $\Delta=0,008$ sec: $K=6,22$,

$$
" \quad A=0,0004, \ldots: K=3,66 \text {. }
$$

Zweiter Teil.

Die Theorie des anomalen Verhaltens der Dielektrika.

1. Zusammenhang der Hauptformen anomalen Verhaltens.

Die im ersten Teile dargestellten Hauptformen des anomalen Verhaltens sind nicht unabhängig voneinander, sondern es läBt sich nachweisen, daB aus dem Restehen der ersten Hauptform, der Rückstandsbildung, das Auftreten von den anderen Hauptformen analogen Erscheinungen notwendig folgt.

Es ist bereits im Abschnitt I, 1 erwälnt worden, daB das zunächst experimentell gefundene Superpositionsprinzip verallgemeinert bei beliebiger zeitlicher Variation der angelegten 
Spannung: $E=\Phi(t)$ zu folgender Formel für den Rückstandsstrom führt:

$$
i_{t}=\int_{-\infty}^{t} d \vartheta \Psi^{\prime}(\vartheta) f^{\prime}(t-\vartheta)
$$

wobei $f(t)$ eine Funktion ist, die den zeitlichen Gang des Rückstandsstromes darstellt, falls zur Zeit $t=0$ an den vorher unendlich lange Zeit der Einwirkung elektrischer Kräfte entzogenen Kondensator plötzlich eine Spannungsdifferenz von der GröBe 1 angelegt wird. Nach den bereits gegebenen Resultaten kann $f(t)$ annähernd dargestellt werden durch

$$
f(t)=\beta . C \cdot t^{-n} \quad(n<1) .
$$

Es soll zunächst der Spezialfall untersucht werden, daB die Spannungsdifferenz der Kondensatorbelegungen eine einfache periodische Funktion der Zeit sei, also

$$
E=E_{0} \sin \frac{2 \pi t}{t} \text {. }
$$

Aus obiger Formel folgt dann:

$$
i_{t}={ }_{\tau}^{2 \pi} E_{0} \int_{-\infty}^{t} d \vartheta \cos \frac{2 \pi \vartheta}{\tau} f(t-\vartheta)
$$

setzt $\operatorname{man}(t-1)=u$, so ergibt sich daraus:

wobei

$$
i_{t}=\frac{2 \pi}{\tau} E_{0}\left\{A \cos \frac{2 \pi t}{\tau}+B \sin \frac{2 \pi t}{\tau}\right\}
$$

$$
\begin{aligned}
& A=\int_{0}^{\infty} f(u) \cos \frac{2 \pi u}{\pi} d u, \\
& B=\int_{0}^{\infty} f(u) \sin \frac{2 \pi u}{t} d u .
\end{aligned}
$$

Dieser Strom $i_{t}$ ist dem normalen Strome übergelagert der sich wieder aus dem normalen Ladungsstrom und der normalen Leitungsstrom zusammensetzt und unter Vernach. lässigung von Selbstinduktion und Widerstand im äußeren die Belegungen verbindenden Kreise dargestellt wird durch

$$
\frac{2 \pi}{r} C E_{0} \cos \frac{2 \pi t}{r}+\frac{4 \pi \lambda}{K} C E \sin \frac{2 \pi t}{t} .
$$


Der anomale, auf Rückstandsbildung beruhende Strom $i_{t}$ besteht somit aus zwei Gliedern, die einfach zum normalen Ladungs- bzw. Leitungsstrom hinzugerechnet werden können und sich durch eine scheinbare Änderung der Normalwerte von Kapazität und Widerstand des Kondensators interpretieren lassen.

Man erhält für die ,scheinbare Kapazität" den Wert:

$$
C^{\prime}=C+A \text {, }
$$

für den reziproken "scheinbaren Widerstand":

$$
L^{\prime}=L+\frac{2 \pi}{x} \cdot B
$$

Führt man für die bisher unbestimmt gelassene Funktion $f(u)$ den experimentell gefundenen Näherungswert

$$
f(u)=\beta \cdot C \cdot u^{-n}
$$

ein, so wird mittels der Substitution $\omega=2 \pi u / \tau$

und :

$$
\begin{aligned}
A & =\left(\frac{\tau}{2 \pi}\right)^{1-n} \cdot \beta C \int_{0}^{\infty} \omega^{-n} \cos \omega d \omega \\
& =\left(\frac{\tau}{2 \pi}\right)^{1-n} \cdot \beta C \cdot \Gamma(1-n) \cos \frac{(1-n) \pi}{2}
\end{aligned}
$$

$$
\begin{aligned}
B & =\left(\frac{\tau}{2 \pi}\right)^{1-n} \cdot \beta C \int_{0}^{\infty} \omega^{-n} \sin \omega d \omega \\
& =\left(\frac{\tau}{2 \pi}\right)^{1-n} \cdot \beta \cdot C \frac{\pi}{2 \Gamma(n) \cos \frac{(1-n) \pi}{2}} .
\end{aligned}
$$

Da $n<1$, also $1-n$ positiv ist, ergibt sich, daB die scheinbare Kapazität $C^{\prime}$ für unendlich rasche Schwingungen den Normalwert $C$ besitzt, bei endlicher Periodendauer $\tau$ vergröbert ist und mit wachsender Periodendauer zunimmt, was mit den im Abschnitt I, 4 mitgeteilten Versuchsergebnissen übereinstimmt.

Analog erhält man für die scheinbare Leitfähigkeit den Ausdruck:

$$
I^{\prime}=L+\frac{2 \pi}{\tau} B=L+\left(\frac{\tau}{2 \pi}\right)^{-n} \beta C \cdot \frac{\pi}{2 \Gamma(n) \cdot \cos \frac{(1-n) \pi}{2}},
$$


d. h. die scheinbare Leitfähigkeit steigt mit abnehmender Periodendauer au. Dementsprechend ist also auch im Dielektrikum eine Wärmeentwickelung zu erwarten, die gröBer ist als die aus dem stationären (wahren) Leitvermögen berechnete und die mit zunehmender Frequenz des Wechselstromes wächst, unter sonst gleichen Bedingungen aber wie die wahre Joulesche Wärme dem Quadrat der Effektivspannung proportional ist. Auch hier besteht also Übereinstimmung mit den im Abschnitt I, 2 besprochenen experimentellen Resultaten.

$\mathrm{DaB}$ bei vorübergehender Verbindung eines Kondensators mit einer Stromquelle konstanter elektromotorischer Kraft (z. B. bei Kapazitätsmessungen mittels des ballistischen Galvanometers) der Rückstandsstrom ebenso wie der normale Leitungsstrom den unmittelbar gefundenen Wert der Kapazität erhöht, und zwar um so mehr, je länger die Ladungszeit war, ist unmittelbar ersicbtlich.

Es sind also Wärmeproduktion im Dielektrikum im Wechselfelde und Abhängigkeit der scheinbaren Dielektrizitätskonstante von Frequenz des Wechselstromes, bzw. von der Ladungszeit bei Gleichstrom notwendige Folgeerscheinungen, wenn Rückstandsbildung vorliegt. Es sind aber nicht auch umgekehrt die ersteren Erscheinungen notwendig auf Rückstandsbildung zurückzuführen, sondern können ohne Rückstandsbildung des Mediums durch andere Ursache (z. B. Leitung) bedingt sein.

Die ponderomotorischen Kräfte, die ein Dielektrikum im relativen Drehfelde erfährt, sind vorläufig nicht berücksichtigt worden. Der gegenseitige Zusammenhang aber von ponderomotorischen Kräften und Wärmeproduktion kann unabbängig von jeder speziellen Hypothese aus allgemeinen energetischen Prinzipien abgeleitet werden.

Erfährt ein ursprünglich ruhendes Dielektrikum im rotierenden Felde ein Drehungsmoment im Sinne der Rotation des Feldes und wird es durch äuBere Kräfte in seiner Ruhelage erhalten, so ist die Arbeit, die das elektrische Drehungsmoment an dem relativ zu ihm rotierenden Dielektrikum leistet, äquivalent der im Dielektrikum entstehenden Wärme. Analog ist bei durch äuBere Kräfte konstant erhaltener Rotation des Dielektrikums im ruhenden Felde die Arbeit dieser äuBeren Kräfte das Äquivalent der entwickelten Wärme. Bezeichnet 
also $W$ die pro Zeiteinheit im Dielektrikum entwickelte Wärme, $D$ das Drehungsmoment der elektrischen Kräfte, $\omega$ die Winkelgeschwindigkeit der relativen Rotation von Feld und Dielektrikum, so können die Größen $W$ und $D$ wechselseitig aus einander bestimmt werden nach der Formel:

$$
w^{\prime}=D . \omega \text {. }
$$

Falls $D$ negativ ist (der Feldrotation entgegengerichtetes Drehungsmoment im Drehfelde, bzw. beschleunigendes Drehungsmoment bei im ruhenden Felde rotierendem Dielektrikum) wird Arbeit gewonnen; das Äquivalent muß dann im verminderten Energieverbrauch in der den stationären Zustand aufrecht erhaltenden Stromquelle liegen.

2. Die Unvereinbarkeit der Anomalien mit der Annahme normalen Verhaltens der Dielektrika.

Es soll nun eingehender untersucht werden, inwieweit die im ersten Teil geschilderten Anomalien wirklich unvereinbar mit den Annahmen der allgemeinen Theorie sind, die ein homogenes isotropes Dielektrikum durch die Angabe von zwei Materialkonstanten: Dielektrizitätskonstante $K$ und spezifisches Leitvermögen $\lambda$ als ausreichend charakterisiert annimmt.

Die Erscheinungen des anomalen Ladungsstromes und der Rückstandsbildung fallen sofort als unvereinbar mit diesen Annahmen heraus.

Bezüglich der Erscheinungen der Energieverluste, der Drehungsmomente im Drehfelde und der Variabilität der scheinbaren Kapazität mit Ladungsdauer oder Periodenzahl ist dies nicht auf den ersten Blick ersichtlich; es wurde oben nachgewiesen, daB in einem Medium, das Rückstandsbildung zeigt, auch diese Erscheinungen auftreten müssen, daB sie aber auch ohne Rückstandsbildung denkbar wären.

a) Die Wärmeproduktion kann einfach als Joule sche Wärme des schwach leitenden Dielektrikums aufgefaBt werden. Viele Beobachter haben sich begnügt, die Abhängigkeit der pro Zeiteinheit entwickelten Wärmemenge $W$ von der Effektivspannung $E$ des Wechselfeldes zu untersuchen und aus dem Resultate, daB $W$ proportional $E^{2}$ sei, auf Leitung als Ursache zurückgeschlossen. Diese Schlußweise ist nicht bindend; 
als Joulesche Wärme müBte die GröBe $W$ unabhängig von der Frequenz des Wechselstromes sein, was tatsächlich nicht der Fall ist, und auch im konstanten elektrischen Felde den. selben Betrag zeigen. Mehrfache Untersuchungen haben aber das Resultat ergeben, daß die aus dem Leitvermögen im stationären Zustande berechnete Joulesche Wärme viel kleiner ist als die im Wechselfelde direkt beobachtete. So finden Moscicki und Altenberg (II, 49), daB bei Glas nur etwa 2 Proz. der im Wechselfelde erzeugten Wärme dem stationären Leitvermögen entsprechen, und Corbino (II, 51), daß die an einem Paraffinpapierkondensator ermittelten Energieverluste einem Widerstande des Dielektrikums von $1400 \Omega$ entsprechen würden, während natürlich der wahre Wert des Widerstandes enorm viel größer ist.

Unter Berücksichtigung der quantitativen Verhältnisse sind disher die beobachteten Energieverluste in Dielektrikas mit der Annahme einer normalen Leitung nicht vereinbar.

b) $\mathrm{DaB}$ ein leitender Körper in einem relativen Drehfelde Drehmomente erfährt, ist bereits von Hertz (III, 1) theoretisch nachgewiesen worden. Verallgemeinerungen dieses Beweises rühren von Heydweiller (III, 3) und Schweidler (III, 5) her. Das Resultat ist folgendes: Rotiert eine Vollkugel rom Radius $R$ und dem spezifischen Leitvermögen (im statischen Maße) $\lambda_{i}$, die von einem Medium mit dem Leitvermögen $\lambda_{\alpha}$ umgeben ist, um eine zu der Richtung eines homogenen elektrischen Feldes von der Stärke $F$ senkrechte Achse mit der konstanten relativen Winkelgeschwindigkeit $2 \pi / \tau$ (also $\tau$ gleich Umlaufsdauer), so ist das Drehungsmoment gegeben durch die Formel:

$$
D=R^{3} F^{2} \frac{\frac{2}{3} \boldsymbol{\tau}\left(\lambda_{a}-\lambda_{z}\right)}{1+\frac{4}{9} \boldsymbol{\tau}^{2}\left(2 \hat{\lambda}_{a}+\lambda_{i}\right)^{2}} .
$$

Das Drehungsmoment ist positiv (beschleunigend im ruhenden Felde, entgegen der Feldrotation gerichtet im Drehfelde), wenn $\lambda_{a}>\lambda_{i}$, negativ (hemmend im ruhenden Felde, im Sinne der Feldrotation im Drehfelde), wenn $\lambda_{i}>\lambda_{a}$; der absoluten Größe nach hängt $D$ auch von der Winkelgeschwindigkeit ab, und zwar derart, daB bei sonst konstanten Verhältnissen einer bestimmten Geschwindigkeit ein Maximum von $D$ entspricht. Qualitativ sind also die beobachteten Formen des Auftretens ponderomotorischer Kräfte im relativen Drehfeld auf 
Leitung zurückzuführen. Zur quantitativen Darstellung erweist sich aber wieder diese Annahme als unzureichend. Die experimentellen Ergebnisse von Arno (II, 7, 15 bis 19, 27) und Threlfall (II, 30, 31) liefern $z$ wischen Drehungsmoment und Feldstärke eine Beziehung: $D$ proportional $F^{n}$, wo $n<2$, statt der theoretischen Beziehung $D$ proportional $\mathbf{F}^{\mathbf{2}}$.

Bei einigen Versuchen v. Langs (II, 52) ist sogar der Sinn der Rotation der umgekehrte, als es nach dem Betrage von $\lambda_{i}-\lambda_{a} \mathrm{zu}$ erwarten wäre; schlecht leitende feste Körper in besser leitenden Flüssigkeiten rotieren bisweilen im Sinne des Drehfeldes statt entgegengesetzt.

c) Daß infolge der Leitung die Dielektrizitätskonstante eines Mediums scheinbar zu groB bestimmt wird, ist ein Umstand, der seit langem bei der Messung von Dielektrizitäts. konstanten berücksichtigt und entweder durch die Wahl der Versuchsanordnung (sehr kurze Ladungszeiten oder sehr schnelle Schwingungen) oder rechnerisch durch separate Bestimmung der Leitung eliminiert wird. Wollte man die im Abschnitt I, 4 angeführten Beispiele auf diese Weise erklären, so würde man auf Werte des spezifischen Leitvermögens der Medien geführt werden, die von anderer Größenordnung sind als die tatsächlich beobachteten. So z. B. erhält man aus den Angaben Beaulards (vgl. p. 721) für Glas den spezifischen Widerstand $\sigma=4.10^{10} \Omega \mathrm{cm}$, während er tatsächlich mindestens zu $10^{14}$ bis $10^{15}$, also rund etwa 10000 mal größer anzunehmen ist.

Obwohl also Erscheinungen von qualitativ gleicher Art wie die beobachteten durch bloBe Leitung entstehen kōnnen, folgt aus den quantitativen Verhältnissen, daB diese Erklärung unzureichend ist. Dies sowie die überhaupt mit der allgemeinen Theorie unvereinbare Rückstandsbildung zwingen also, in irgend welcher Weise die Anomalien durch modifizierte Annahmen in die Theorie einzufügen.

\section{Anomalien der Struktur (Inhomogenität).}

Die erste Möglichkeit, die Anomalien theoretisch zu begründen, liegt darin, zwar die allgemeinen Grundannahmen der Theorie unverändert beizubehalten, aber jene Medien, die Anomalien zeigen, als nicht homogen aufzufassen. 
In der Tat hat Maxwell (I, 5) gezeigt, daß ein ,ge schichtetes" Dielektrikum, dessen einzelne Schichten sich durch aus normal verhalien, bei dem aber der Quotient $\lambda / K$ nich den gleichen Wert in jeder Schichte besitzt, als Ganzes dir Erscheinungen der Rückstandsbildung zeigen müsse. Er ha ferner darauf hingewiesen, daB bei nicht blätteriger Struktur sondern beliebiger räumlicher Anordnung der nicht gleich artigen Bestandteile des Mediums analoge Resultate sich er geben. Weitere Ausführungen dieser Maxwellschen Theorif der geschichteten Dielektrika finden sich in den Arbeiten vor Hess (I, 24) und Houllevigue (I, 33).

In der Maxwellschen Darstellung werden folgende GröBer als Funktionen der Materialkonstanten und der Anfangs. bedingungen explizit berechnet:

1. Die "disponible Ladung"; 2. der stationäre Leitungsstrom nach unendlich langer Einwirkung einer konstanten elektromotorischen Kraft; 3. der zeitliche Verlauf der freien Rückstandsladung eines Kondensators, dessen Belegungen nach unendlich langdauernder Ladung durch eine konstante elektro. motorische Kraft plötzlich entladen und hierauf voneinander isoliert werden; 4. die gesamte Elektrizitätsmenge (gesamte Rückstandsladung), die einen nach unendlicher Ladungsdauer angelegten KurzschluB zwischen beiden Belegungen durch. flieBt.

Der Rückstandsstrom bei. konstanter elektromotorischer Kraft als Funktion der Zeit wurde von Maxwell nicht berechnet. Dieses Problem führt auf groBe mathematische Schwierigkeiten und ist daher im folgenden nur so weit ausgeführt, daB die Gültigkeit des Superpositionsprinzips theoretisch abgeleitet werden kann.

Die Voraussetzungen der folgenden Ableitung sind gezenüber denen Maxwells einerseits eingeschränkt durch die Annahme, daß die Dielektrizitätskonstante $K$ des inhomogenen Mediums durchwegs konstant sei, andererseits erweitert durch die Annahme, daB bei Fortschreiten in einer bestimmten Richtung (der $X$-Achse) das Leitvermögen $\lambda$ nicht sprungweise eine endliche Anzahl von Änderungen, den Werten $\lambda_{1}$ bis $\lambda_{n}$ entsprechend, sondern kontinuierlich sich ändere, also durch eine beliebige stetige Funktion $\lambda(x)$ darstellbar sei. 
Es sei also gegeben eine unendlich ausgedehnte Platte eines Dielektrikums ron der Dicke $l$, die zwischen zwei metallischen Belegungen mit konstanten Potentialen $V=E$ und $V=0$ sich befinde.

Die Dielektrizitätskonstante habe den konstanten Wert $K$, das spezifische Leitvermögen sei eine gegebene Funktion $\lambda(x)$.

Für einen beliebigen Punkt im Dielektrikum gelten dann die Differentialgleichungen:

$$
\begin{aligned}
& \frac{\partial X}{\partial x}=\frac{4 \pi}{K} \varrho, \\
& \frac{\partial \varrho}{\partial t}=-\frac{\partial}{\partial x}[\lambda(x) X],
\end{aligned}
$$

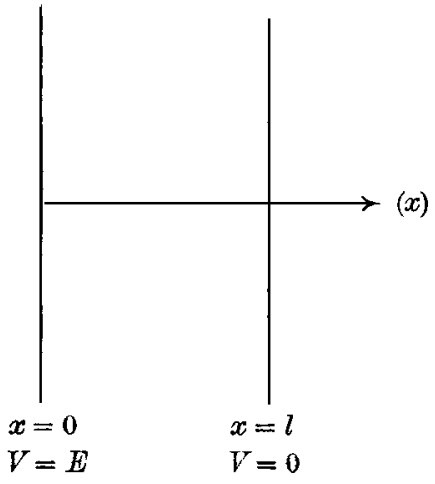

Fig. 1.

wobei $X$ die elektrische Feldstärke, $\varrho$ die Raumdichte der wahren Ladung bezeichnet.

Daraus erbält man für die Feldstärke $X$ die Differentialgleichung:

$$
\frac{K}{4 \pi} \frac{\partial^{2} X}{\partial x \partial t}=-\frac{\partial[\lambda(x) X]}{\partial x}
$$

und durch Integration:

$$
\frac{K}{4 \pi} \frac{\partial X}{\partial t}+\lambda(x) X=\psi(t) .
$$

$\psi(t)$ ist zunächst eine als Integrationskonstante bei der Integration nach $x$ auftretende willkürliche Funktion der Zeit, die aber dadurch bestimmt ist, daB zu jeder Zeit die Bedingung erfüllt sein muB:

Da

$$
\int_{0}^{\ell} X d x=E .
$$

$$
\frac{\partial E}{\partial t}=0=\int_{0}^{l} \frac{\partial X}{\partial t} d x,
$$

erhält man aus Gleichung (4):

$$
\psi(t)=\frac{1}{l} \int_{0}^{l} \lambda(x) X d x .
$$


Diese Funktion $\psi(t)$ hat eine einfache physikalische Bedeutung: sie ist der Gesamtstrom pro Flächeneinheit in der Richtung der positiven $x$-Achse; in Gleichung (4) ist die Zerlegung in einen Verschiebungsstrom $\frac{\pi}{4 \pi} \frac{\partial X}{\partial t}$ und einen Leitungsstrom $\lambda(x) X$ angedeutet.

Gleichung (4) liefert nach abermaliger Integration die Formel ${ }^{1}$ ):

$$
\left\{\begin{aligned}
X & =e^{-\frac{4 \pi}{K} \lambda(x) t}\left\{\frac{E}{l}+\int_{0}^{t} \psi(t) e^{+\frac{4 \pi}{K} \lambda(x) t} d t\right\} \\
& =e^{-\frac{4 \pi}{K} \lambda(x) t}\left\{\frac{E}{l}+\int_{0}^{t} d t\left[e^{+\frac{4 \pi}{K} \lambda(x) t} \cdot \frac{1}{l} \int_{0}^{t} \lambda(x) X d x\right]\right\} .
\end{aligned}\right.
$$

Durch diese Integralgleichung ist die Feldintensität $X$ als Funktion ron $x$ und $t$ bestimmt; eine explizite Darstellung von $X$ oder $\psi(t)$ daraus zu gewinnen, ist dem Verfasser nicht gelungen. Immerhin genügen die erhaltenen Resultate zum Nachweis des Superpositionsprinzips.

Aus den Gleichungen (4) und (6) folgt, daß bei konstanter Potentialdifferenz die Differentialgleichung gilt:

$$
\frac{\pi}{4 \pi} \cdot \frac{\partial X}{\partial t}+\lambda(x) X=\frac{1}{l} \int_{0}^{l} X \lambda(x) d x .
$$

Wenn also an einem Kondensator, für den $K, l$ und $\lambda(x)$ gegeben sind, in zwei Versuchen bloß die Anfangsbedingungen für $t=0, X=X_{0}(x)$ und $X^{\prime}=X_{0}^{\prime}(x)$ sowie die GröBen $E$ und $E^{\prime}$ verschiedeu sind, so ist in beiden Fällen obige Differentialgleichung erfüllt. Setzt man daher $X_{t}^{\prime}=X_{t}+\Delta_{t}$, so gilt auch für die Differenz $\Delta_{t}$ die Gleichung:

$$
\frac{K}{4 \pi} \frac{\partial \Delta_{t}}{\partial t}+\lambda(x) \Delta_{t}=\frac{1}{l} \int_{0}^{l} \Delta_{t} \cdot \lambda(x) d x,
$$

1) Anmerkung: Die Formel (7) enthält in der ursprünglichen Publikation (Wiener Sitzungsber. 116. p. 1075) zwei Druckfehler, indem in der ersten Zeile $d x$ statt $d t$, in der zweiten Zeile

eingesetzt ist.

$$
\int_{0}^{l} X d x \text { statt } \frac{1}{l} \int_{0}^{l} \lambda(x) X d x
$$


d. h. der zeitliche Verlauf der Änderung der Feldintensität $X^{\prime}$ und damit auch der diese Änderung begleitende Rückstandsstrom kann aufgefaßt werden als Superposition der beiden Vorgänge, die sich abspielen würden, wenn zur Zeit $t=0$ einmal die momentane Verteilung der elektrischen Kraft durch $X_{0}$. das andere Mal durch $\Delta_{0}$ gegeben wäre. Wird also in einem beliebigen Stadium der Rückstandsbildung die Potentialdifferenz der Belegungen plötzlich um den Betrag $\Delta E$ und damit die Feldstärke überall um den Betrag $\Delta E / l$ geändert, so ist der weitere Ablauf des Rückstandsstromes gegeben durch die Superposition jenes Stromes, der ohne Änderung der Potentialdifferenz weiter erfolgt wäre, und eines Rückstandsstromes, der durch Anschaltung der Spannung $\Delta E$ an den vorher unendlich lange Zeit ungeladenen Kondensator erzeugt worden wäre.

Die Maxwellsche Theorie der inhomogenen Dielektrika gibt also Aufschluß über die wesentlichsten Eigenschaften, die an den anomalen Dielektrikas tatsächlich vorgefunden werden. Die explizite Darstellung der experimentell beobachtbaren Größen führt aber auf mathematische Schwierigkeiten, so daß eine Prüfung der Theorie durch Vergleich mit der Erfahrung bezüglich der quantitativen Verhältnisse nicht ausgeführt werden kann.

\section{Anomalien der Leitung.}

Fine zweite Möglichkeit der theoretischen Darstellung der Erscheinungen an Dielektrikas besteht darin, die Annahme eines konstanten spezifischen Leitvermögens fallen zu lassen und zu ersetzen durch eine solche, die kompliziertere Formen des Leitungsvorganges voraussetzt. Insbesondere in Anlehnung an die bei ionisierten Gasen beobachteten Erscheinungen können die Gesetze der Ionenleitung auf flüssige und feste Dielektrika übertragen werden.

Die Theorie der Elektrizitätsleitung in ionisierten Gasen geht ron folgenden Voraussetzungen aus:

Durch Wirkung eines „Ionisators" entstehen pro Volumund Zeiteinheit $q$ Ionenpaare; ein Teil der Ionen verschwindet durch Wiedervereinigung zu neutralen Molekülen (,Molisierung("), und zwar ist dieser Betrag, pro Volum- und Zeiteinheit gerechnet, gegeben durch $\alpha n_{1} n_{2}$, wo $n_{1}$ und $n_{2}$ die 
Zahlen der in der Volumeinheit vorhandenen positiven bzw negativen Ionen, $\alpha$ ein für eine bestimmte Gattung von Ionen paaren charakteristischer sogenannter „Wiedervereinigungء koeffizienten" ist. Es gilt also die Gleichung:

$$
\frac{d n}{d t}=q-\alpha n_{1} n_{2} \text {. }
$$

In einem elektrischen Felde ist in dieser Gleichung nocl ein Glied einzufügen, daß die Differenz der Zahlen der durcl den Strom zu- und abgeführten Ionen angibt, also:

$$
\begin{aligned}
& \frac{d n_{1}}{d t}=q-\alpha n_{1} n_{2}-s_{1}, \\
& \frac{d n_{2}}{d t}=q-\alpha n_{1} n_{2}-s_{2} .
\end{aligned}
$$

Die positiven bzw. negativen Ionen bewegen sich nun ir einem elektrischen Felde mit einer der Feldintensität propor. tionalen Geschwindigkeit $u_{1}=c_{1} X, u_{2}=-c_{2} X$, worin $c_{1}$ und $c_{\varepsilon}$ die sogenannten spezifischen Geschwindigkeiten oder Beweglich. keiten der beiden Ionenarten sind.

Daraus ergeben sich für den Fall der Elektrizitätsleitung in einem von zwei unendlich ausgedehnten parallelen Platten (in der Distanz l) begrenzten ionisierten Gase - bei konstanter Potentialdifferenz $E$ der beiden Platten - folgende Gleichungen :

$$
\begin{aligned}
& {\left[n_{1}-n_{2}\right] \varepsilon=-4 \pi \frac{\partial X}{\partial x},} \\
& \partial n_{1}=q-c n_{1} n_{2}-\frac{\partial}{\partial x}\left[n_{1} c_{1} X\right], \\
& d t=q n_{1} n_{2}+\frac{\partial}{\partial x}\left[n_{2} c_{2} X\right], \\
& \frac{\partial n_{2}}{\partial t}=q-\epsilon n^{-} \\
& \int_{U}^{l} X d x=E .
\end{aligned}
$$

Die allgemeine Integration dieses Gleichungssystems ist bisher nicht durchgeführt (vgl. J. J. Thomson, Conduction of electricity through Gases p. 64-73, Cambridge 1903); doch sind folgende Resultate leicht ersichtlich:

Durch Einschalten eines elektrischen Feldes wird der ursprüngliche Ionengehalt $n_{0}=\sqrt{q / \alpha}$ verringert, die Stromstärke nimmt mit der Zeit $a b$ und erreicht einen stationären 
Grenzwert, der bei Anwendung verschiedener Werte der elektromotorischen Kraft nicht dieser proportional ist; das spezifische Leitvermögen eines ionisierten Gases ist also nach StromschluB zeitlich variabel und im stationären Endzustand eine Funktion der Stromdichte, und zwar eine mit steigender Stromdichte abnehmende.

Die zeitliche Variation erfolgt in Gasen sehr schnell, so daß sie in den meisten Fällen experimentell nicht verfolgt werden kann; die Abhängigkeit des stationären Leitvermögens von der Stromdichte bzw. von der elektromotorischen Kraft zeigt sich in bekannter Weise durch die "Charakteristik", d. i. die Kurve $i=f(E)$, welche zu der Unterscheidung ,Ohmscher Strom" (dem Ohmschen Gesetz gehorchender Strom) für kleine Feldintensitäten oder Spannungen, ,unvollständig gesättigter Strom" für mittlere und "Sättigungsstrom" (von der elektromotorischen Kraft unabhängiger konstanter Strom) für große Feldintensitäten oder Spannungen gefübrt hat.

Ferner folgt aus den Gleichungen, daB im stationären Zustande die Ionenzahl pro Volumeinheit nicht mehr räumlich konstant ist: in den den Elektroden benachbarten Schichten sind Ionen des einen (und zwar dem der Elektrode entgegengesetzten) Vorzeichens im Überschusse vorhanden; dadurch ist auch das ursprünglich Lomogene elektrische Feld gestört, in der Nähe der Elektroden über den Mittelwert erhöht, in der Mitte unter denselben erniedrigt.

Es ist ein naheliegender Gedanke, diese Annahmen auch auf flüssige und feste Dielektrika zu übertragen, die Leitung in diesen Medien als Tonenleitung aufzufassen.

Einige anomale Erscheinungen bei der Leitung flüssiger Dielektrika (vgl. im Literaturverzeichnis Abteilung IV), so die zeitliche A bnahme der Stromstärke und die Nichtproportionalität von Stromstärke und elektromotorischer Kraft im stationären Zustande, wurden auch bereits in dieser Weise gedeutet, ja an einigen flüssigen Dielektrikas wurden der GröBenordnung nach Ionenbeweglichkeiten und Ionenzahlen bestimmt (Schweidler, IV, $7,8,10)$.

Aber auch die Erscheinungen der Wärmeproduktion, der Rotationen und der Rückstandsbildung lassen sich qualitativ auf diesem Wege ableiten. 
Bezüglich der Wärmeproduktion, von der oben nachgewiesen wurde, daB sie nicht als die einem konstanten Leitvermögen entsprechende Joulesche Wärme gedeutet werden könne, ist zunächst ersichtlich, daB bei Anwendung alternierender Felder (Wechselstrom) nicht der infolge des Stromdurchganges verringerte Wert des Leitvermögens im stationären Zustande, sondern jener Wert einzusetzen ist, der kurz nach StromschluB vorhanden ist; es wird damit auch begreiflich, daB das mittlere Leitvermögen abhängig ist von der Dauer der Einwirkung des Feldes in bestimmter Richtung, dab mit abnehmender Periodendauer des Wechselstromes das Leitvermögen immer näher an den — im feldlosen Raume gültigen Wert -

heranrückt.

$$
\left(c_{1}+c_{2}\right) \varepsilon n_{0}=\left(c_{1}+c_{2}\right) \varepsilon \sqrt{\frac{q}{\alpha}}
$$

Bezüglich der Rotationserscheinungen und Drehungsmomente, die nach der im Abschnitt II, 2 angegebenen Formel auf die Leitungskonstanten zurückführbar sind, gilt dasselbe. Insbesondere wird es verständlich, daB das Drehungsmoment $D$ langsamer wächst als $F^{2}$, da das spezifische Leitvermögen mit steigender Feldintensität abnimmt.

Auch die Rückstandsbildung im engeren Sinue kann zurückgeführt werden auf die durch die Stauung der Ionen in der Nähe der Elektroden bedingten Feldstörungen. Es läBt sich hier einfach die im vorigen Abschnitt behandelte $\mathrm{Maxwellsche}$ Theorie der geschichteten Dielektrika anwenden:

Ein Medium mit Ionenleitung ist zwar nicht von vornherein inhomogen, aber es wird inhomogen bezüglich seiner Leitfähigkeit infolge des Stromdurchganges; die ursprüngliche Leitfähigkeit im feldlosen Raume wird - wie oben erwähnt verändert, und zwar in der Nähe der Elektroden stärker erniedrigt als in der Mitte. Somit muB ein derartiges Medium nach einiger Dauer der Einwirkung des elektrischen Feldes das Verhalten eines geschichteten Dielektrikums zeigen.

Wenn so die Annabme von Ionenleitung auch in flüssigen und festen Dielektrikas genügt, die anomalen Erscheinungen in qualitativer Hinsicht zu erklären, so ergeben sich doch Bedenken bei der Anwendung dieser Theorie zur Darstellung der quantitativen Verhältnisse. 
Zunächst sind, wie oben erwähnt, Schwierigkeiten mathematischer Natur vorhanden, welche die exakte Lösung des Problems verhindern: in einem Medium, dessen Ionenkonstanten (Ionisierungsstärke $q$, Koeffizient der Wiedervereinigung $\alpha$, Beweglichkeiten $c_{1}$ und $c_{2}$ ) gegeben sind, den Strom als Funktion der Zeit und der elektromotorischen Kraft darzustellen. Diese Schwierigkeiten nun beeinträchtigen wohl die Anvendbarkeil der Theorie, ohne natürlich gegen ihre Richtigkeit etwas zu beweisen.

Aber auch in bezug auf die Richtigkeit ergeben sich Bedenken. Wäre die Abnahme des Rückstandsstromes von seinem Anfangswerte auf einen hierzu relativ kleinen Endwert bedingt durch die Entionisierung infolge des Stromdurchganges, so müBte man annehmen, daB der stationäre Strom der unvollkommen oder vollkommen gesättigten Phase angehört (vgl. Fig. 2).

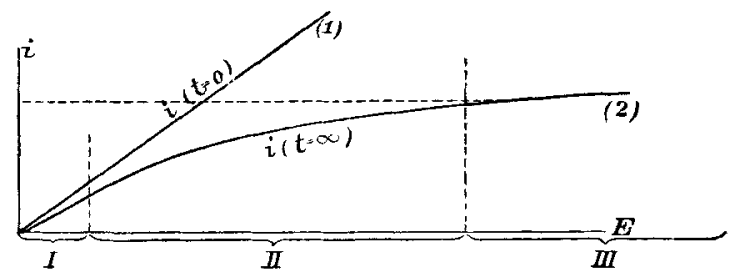

Fig. 2.

I. Phase: Ohmscher Strom.

II. Phase: Unvollständig gesättigter Strom.

III. Phase: Sättigungsstrom.

Es zeigt sich aber tatsächlich, daB der stationäre Endwert des Stromes in einem rückstandbildenden Dielektrikum (z. B. Glas) innerhalb weiter Grenzen dem Ohmschen Gesetze entspricht, d. h. der elektromotorischen Kraft proportional ist (man vergleiche die experimentellen Resultate von E. Warburg, Wied. Ann. 21. p. 622, und F. M. Exner, Verh. d. D. Phys. Ges. 3. p. 26. 1901, sowie E. v. Schweidler (I, 46)). Für den "Ohmschen Strom" (Phase I) aber ist wiederum die Differenz zwischen dem Anfangswerte des Stromes für $t=0$ (in der Figur durch die Gerade 1 dargestellt) und dem stationären Endwerte (in der Figur Kurve 2) gering. 
Die Annahme bloB einer Gattung von Ionenpaaren ist also nicht zulässig.

Man könnte nun annehmen, daß von jedem Vorzeichen Ionen mit sehr verschiedenen Werten der spezitischen Geschwindigkeit gleichzeitig im Dielektrikum vorhanden seien, so daB bei bestimmter elektromotorischer Kraft für die eine Gattung (die leicht beweglichen) bereits Sättigungsstrom eintritt, während für die anderen (schwer beweglichen) noch Ohmscher Strom herrscht. Aber auch diese kompliziertere Annahme ist vollkommen unvereinbar mit folgender Tatsache:

Nach Einschaltung einer elektromotorischen Kraft vom Werte $E_{1}$ zur Zeit $t=0$ beobachtet man einen Strom, darstellbar durch $i_{1}=a_{1}+f^{\prime}(t)$, wobei $f^{\prime}(t)$ von einem hohen Anfangswerte asymptotisch auf Null absinkt und $a_{1}$ den stationären Endwert darstellt; wird nach hinreichend langer Zeit T, so daß $f(T)$ praktisch gleich Null gesetzt werden kann, die elektromotorische Kraft plötzlich vergröBert, z. B. auf den Wert $E_{2}=k E_{1}$, so beobachtet man tatsächlich einen Strom $i_{2}$, der als Funktion der Zeit gegeben ist durch die Formel:

$$
i_{2}=k a_{1}+(k-1) f(t-T),
$$

entsprechend der früher erwähnten Proportionalitätsbeziehung zwischen stationärem Teil der Strömung und elektromotorischer Kraft, sowie dem Superpositionsprinzip für den zeitlich variablen Bestandteil des Stromes. Da $f(0)$ groB gegen $a_{1}$ ist, setzt also unmittelbar nach del VergröBerung der elektromotorischen Kraft der Strom $i_{2}$ mit einem Werte ein, der nahezu $(k-1)$ mal größer ist als der Anfangswert von $i_{1}$ für $t=0$.

Der auf Ionenleitung beruhende Strom könnte aber unmittelbar nach Einschaltung der elektromotorischen Kraft $E_{2}$ höchstens den Wert $k a_{1}$ erreichen, da ja die Leitfähigkeit des Mediums durch den langdauernden Strom $i_{1}$ bereits auf den mittleren Betrag von $a_{1} / E_{1}$ herabgedrückt wurde.

Es ist also resumierend über die Anwendung der Theorie der Ionenleitung auf flüssige und feste Dielektrika zu sagen, $\mathrm{dab}$ die von ihr geforderten anomalen Eigenschaften des spezifischen Leitvermögens (zeitliche Variabilität, Abhängigkeit von Stromdichte) zwar an den tatsächlich beobachteten Erscheinungen mitbeteiligt sein können, daß sie aber für sich 
allein nicht ausreichend ist, die wesentlichen Eigentümlichkeiten der beobachteten Phänomene, vor allem die Gesetze der Rückstandsbildung zu erklären.

\section{Anomalien des dielektrischen Verhaltens.}

Eine dritte Möglichkeit der theoretischen Behandlung liegt in der Annahme von Anomalien des dielektrischen Verhaltens. An Stelle der Voraussetzung, daB die dielektrische Verschiebung (oder Polarisation) zu jeder Zeit der elektrischen Feldstärke proportional sei und daB dieser Proportionalitätsfaktor eben durch den konstanten Wert der Dielektrizitätskonstante gegeben sei, treten kompliziertere Bedingungen, nach denen der jeweilige Wert des Verschiebungsvektors $D$ nicht allein eine Funktion des simultanen der Feldstärke $\mathfrak{E}$, sondern auch der vorhergegangenen Zustände des Mediums sein soll. Mit anderen Worten: analog wie in bezug auf Elastizität und Magnetismus zeigen viele Medien auch in bezug auf dielektrische Vorgänge Nachwirkungserscheinungen oder Hysteresis im weiteren Sinne des Wortes.

Allgemein gehaltene Sätze analogen Inhaltes sind oftmals ausgesprochen worden, doch nur wenige Versuche liegen vor, die funktionelle Beziehung zwischen Momentanwert der Verschiebung und der "Vorgeschichte" des Mediums präzise zu formulieren und damit das Fundament für eine eigentliche Theorie der Hysteresiserscheinungen zu schaffen.

a) Hysteresis im engeren Sinne.

Unter Hysteresis im engeren Sinne (,,hysteresis proprement dit") bezeichnen manche Autoren, speziell Beaulard (II, 37) jene Form der Nachwirkungserscheinungen, wie sie an ferromagnetischen Substanzen, besonders an weichem Eisen, experimentell gründlich untersucht und auch theoretisch bis zu einem gewissen Grade befriedigend dargestellt wurden (vgl. Winkelinann, Handbuch der Physik, Bd. V, 1, p. 217-222). Es ist naleliegend, die Gesetze der magnetischen Hysteresis einfach auf die dielektrische zu übertragen: bei variabler Feldintensität ist die einem bestimmten Werte derselben zugeordnete Verschiebung gröber bei abnehmendem als bei zunehmendem Gange der Feldintensität; infolgedessen tritt bei zyklischer 
Elektrisierung (Wechselspannung) in der graphischen Darstellung eine geschlossene Kurve auf (Hysteresisschleife), deren Flächeninhalt bekanntlich die pro Periode im Wärme umgesetzte Energie angibt. Außerdem führt diese Anschauung zu den Begriffen "dielektrische Koerzitivkraft" (durch $O A$ ) und

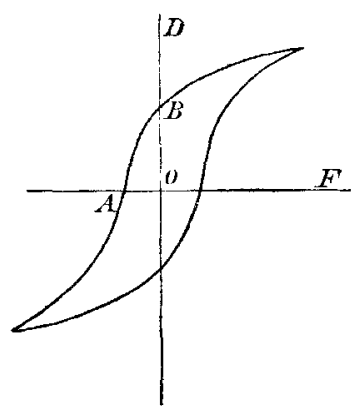

Fig. 3. "remanente Elektrisierung" (durch $O B$ dargestellt).

Für die Abhängigkeit der Hysteresisarbeit von der Amplitude der periodischen Magnetisierung haben die Versuche im allgemeinen eine Formel ergeben von der Form:

$$
W^{r}=\eta . H^{n} \text {, wo } n=\text { ca. } 1,6 \text {. }
$$

Die Übereinstimmung der von Arno (l. c.) gefundenen Beziehung zwischen der elektrischen Hysteresisarbeit und der Effektivspannung ( $W^{\prime}$ proportional $E^{n}$, wo $n$ zwischen 1,5 und 1,96 ) mit obiger Formel wurde oftmals als Beweis für die Analogie der magnetischen und der dielektrischen Hysteresis betrachtet.

Insbesondere Beaulard (II, 37) hat die Unanwendbarkeit dieser Theorie nachgewiesen. Bei der magnetischen Hysteresis ist die Amplitude der periodisch wechselnden magnetischen Feldintensität $\mathfrak{Y}$ in erster Linie maßgebend, die Periodendauer von minder wesentlichem und noch nicht ganz sicher festgestelltem EinfluB. Die Abhängigkeit der dielektrischen „Hysteresisarbeit" von der Dauer der Periode (vgl. Abschnitt I, 2 und 3) stört die Analogie bedeutend.

Ferner ist darauf hinzuweisen, daB "Remanenz" und „Koerzitivkraft" auf elektrischem Gebiete noch nicht experimentell sichergestellt werden konnten (Germanischskaja, VI, 3) und endlich, daB die Rückstandserscheinungen, bei denen der zeitliche Verlauf des anomalen Stromes das erste Problem bildet, in dieser der magnetischen Theorie nachgebildeten überhaupt nicht darstellbar sind.

b) Viskose Hysteresis.

$b_{1}$ ). Auf Grund der oben angeführten Überlegungen hat eine Reihe von Forschern die Theorie der elektrischen Hysteresis 
im engeren Sinne - analog der magnetischen Hysteresis - verworfen und "viskose Hysteresis" (auch ,elektrische Viskosität" genannt) angenommen (Schaufelberger, II, 32, 34; Beaulard, II, 36, 37; Arno in seinen späteren Arbeiten, II, 27; Porter und Morris, II, 22 u. a.).

Hierunter versteht man die Tatsache, daB bei plötzlicher Erregung eines elektrischen Feldes die dielektrische Verschiebung nicht momentan den durch $K(F$ gegebenen Wert annimmt, sondern ihn erst allmählich ansteigend asymptotisch erreicht. Bei kontinuierlicher (eventuell periodischer) Änderung des Feldes $\&$ bleibt daher die Verschiebung $\mathfrak{D}$ stets hinter ihrem „Sollwerte“ zeitlich zurück.

Diese, als ,dielektrische Nachwirkung“ auch auf dem Gebiete der Rückstandsbildung versuchte Annahme (Boltzmann, Romich und Nowack, I, 7; Hopkinson, I, 9-11; Wüllner, I, 12 u. a.) erfordert nun eine schärfere Fassung.

\section{$b_{2}$ ) Pellats Theorie.}

Die einzige bisher tatsächlich erfolgte präzise Formulierung rührt von Pellat her (I, 39, 41 identisch mit II, 33, 39). Der Grundgedanke ist folgender:

Wird an einer Stelle eines dielektrischen Mediums, das hinreichend lange Zeit der Einwirkung eines elektrischen Feldes entzogen war, plötzlich zur Zeit $t=0$ ein Feld von der Intensität $F_{0}$ erzeugt und für $t>0$ konstant erhalten, so nimmt die Verschiebung $\mathfrak{D}$ ebenfalls plötzlich zur Zeit $t=0$ den Wert $K \Theta_{0}$ an, steigt aber dann allmählich an nach der Formel:

$$
\mathfrak{D}_{b}=K \mathbb{E}_{0}+\left(1-e^{-a t}\right) \varepsilon K \xi_{0},
$$

wo $\alpha$ und $\varepsilon$ zwei Materialkonstanten sind.

Es wird für

$$
t=\infty, \mathfrak{D}_{t}=\mathfrak{D}_{\infty}=(1+\varepsilon) K \mathfrak{E}_{0} .
$$

Somit gilt allgemein die Gleichung:

$$
\frac{d \mathfrak{D}_{t}}{d t}=\alpha e^{-a . t} \cdot \varepsilon K \mathfrak{E}_{0}=-\alpha\left(\mathfrak{D}_{t}-\mathfrak{D}_{\infty}\right),
$$

d. h, die Verschiebung strebt einem stationären Endwerte $\mathfrak{D}_{\infty}$ zu und ihre Änderungsgeschwindigkeit ist jederzeit proportional der Differenz dieses Endwertes und des momentanen Wertes. 
Bei beliebiger zeitlicher Variation der Feldintensität $\mathbb{E}_{t}=f(t)$ gilt ebenfalls die Differentialgleichung:

$$
\frac{d \mathfrak{D}_{t}}{d t}=K \frac{d \mathfrak{E}_{t}}{d t}+\alpha\left[\varepsilon K^{r} \tilde{E}_{t}-\mathfrak{D}_{t}\right]
$$

oder wenn man $\mathscr{D}_{t}=K \mathfrak{F}_{t}+\mathscr{D}_{t}^{\prime}$ setzt:

$$
\frac{d \mathfrak{D}_{t}^{\prime}}{d t}=\alpha\left[K \mathbb{F}_{t}-\mathscr{D}_{t}\right] \text {. }
$$

Pellat bezeichnet die Größe $K$ als ,wahre Dielektrizitätskonstante", $K \mathbb{E}$ als ,fiktive Polarisation" und $\mathfrak{D}^{\prime}$ als „wahre Polarisation".

An Stelle dieser - vom Standpunkte der Maxwellschen Theorie aus etwas willkürlich gewählten - Bezeichnungsweise Pellats soll im folgenden $K \mathbb{E}$, ,normale Verschiebung" und $\mathfrak{D}^{\prime}$ „,viskose Verschiebung“ genannt werden.

Auf Grund obiger Annahmen ist also ein Dielektrikum im allgemeinen durch vier Materialkonstanten zu charakterisieren: zu Dielektrizitätskonstante $K$ und spezifischem Leitvermögen $\lambda$ treten noch die Größen $\varepsilon$ und $\alpha ; \varepsilon$ ist von der Dimension einer reinen Zahl und gibt das Verhältnis des stationären Endwertes der viskosen Verschiebung zu der normalen Verschiebung an; $\alpha$ ist von der Dimension einer reziproken Zeit und $1 / \alpha$ bedeutet die Relaxationszeit der viskosen Verschiebung.

Es ergeben sich ferner folgende Konsequenzen:

1. Bei konstanter Feldintensität $\xi_{0}$ ist

$$
\mathfrak{D}=K\left(\mathfrak{E}_{0}+\left(1-e^{\alpha t}\right) \varepsilon K \mathfrak{E}_{0}\right.
$$

und daher der Verschiebungsstrom pro Flächeneinheit

$$
y_{1}=-\frac{1}{4 \pi} \cdot \frac{d \mathfrak{D}}{d t}=\frac{\alpha}{4 \pi} e^{-\alpha t} \varepsilon K \mathfrak{E}_{0} .
$$

Ein diesem Verschiebungsstrom entsprechender Leitungsstrom zirkuliert in der Zuleitung. Hat das Dielektrikum eines Kondensators die FlächengröBe der Belegung $f$ und die Dicke $d$, so daB die Potentialdifferenz der Belegungen gegeben ist durch $E=d . \varepsilon_{0}$, so gilt für den Gesamtstrom in der Zuleitung:

$$
J=\alpha \varepsilon C E, e^{-\alpha t} \text {. }
$$

Wird der Kondensator nach unendlich langer Ladungsdauer entladen, indem zur Zeit $t=0$ die Belegungen ohne 
Einschaltung einer elektromotorischen Kraft direkt leitend verbunden werden, so ist

$$
\begin{aligned}
& \mathcal{D}=\varepsilon K \mathfrak{E}_{0} e^{-\alpha t}, \\
& y_{3}=-\frac{1}{4 \pi} \frac{d \mathscr{D}}{d t}=--_{4 \pi}^{\alpha} e^{-a t} \varepsilon K \mathfrak{E}_{0}=-y_{1},
\end{aligned}
$$

d. h. der durch „Freiwerden des Rückstandes" entstandene Strom $y_{2}$ ist dem rückstandsbildenden Strom" $y_{1}$ entgegengesetzt gleich. Wird der Kondensator nach der endlichen Ladungsdauer $T$ entladen, so ist

$$
\begin{gathered}
\text { für } t=0 ; \mathfrak{D}=\varepsilon K\left(\mathfrak{F}_{0}\left(1-e^{-\alpha T}\right),\right. \\
\text { für } t>0 ; \mathfrak{D}=\varepsilon K \mathbb{E}_{0}\left(1-e^{-\alpha}\right) e^{-\alpha t}, \\
y_{2}=\frac{\alpha}{4 \pi} \varepsilon K\left(\mathfrak{E}_{0}\left(1-e^{-\alpha T}\right) e^{-\alpha t}=\frac{\alpha \varepsilon K \xi_{0}}{4 \pi}\left[e^{-\alpha t}-e^{-\alpha(t+T)}\right],\right.
\end{gathered}
$$

d. h. der nun auftretende Strom entspricht dem Superpositionsprinzip.

Qualitativ sind also die empirischen Gesetze der Rückstandsbildung aus der Pellatschen Theorie abzuleiten; in quantitativer Beziehung besteht Nichtübereinstimmung bezüglich der Form des zeitlichen Verlaufes, für den die Theorie eine Exponentialfunktion $e^{-\alpha t}$, die Erfahrung die Formel $B t^{-n}$ liefert. Pellat meint, die Versuche J. Curies zitierend (I, 21), daB die Beobachtung nicht hinreichend genau sei, um die Differenz $z$ wischen der theoretischen und der empirischen Formel mit Sicherheit konstatieren zu können. Dies ist wohl ein Irrtum; auBer J. Curies haben auch die ron Pellat nicht zitierten Experimentaluntersuchungen von Kohlrausch (I, 1), Hopkinson (I, 9, 10, 11), Giese (I, 15), Dieterici (I, 18) u. a. das gleiche Resultat ergeben, und Beobachtungsfehler von der GröBe der Differenz der nach beiden Formeln berechneten Stromstärken sind schlechthin ausgeschlossen.

Bezüglich der Gesetze der Rückstandsbildung bedarf also die Pellatsche Theorie jedenfalls einer Modifikation.

2. Für den Fall eines sinusförmigen Wechselfeldes

$$
\mathfrak{E}=\mathfrak{E}_{0} \sin \frac{2 \pi t}{\tau}
$$

lassen sich die Erscheinungen übersehen, indem man in die im zweiten Teile, Abschnitt 1, angeführten Formeln (p. 722 
und 723) für die unbestimmt gelassene Funktion $f(u)$ auf Grund der eben abgeleiteten Resultate setzt:

$$
f(u)=a \varepsilon C \cdot e^{-a u} \text {. }
$$

Man erhält also:

wobei

$$
i=\frac{2 \pi E_{0}}{r}\left\{A \cos \frac{2 \pi t}{\tau}+B \sin \frac{2 \pi t}{\tau}\right\}
$$

$$
\begin{aligned}
& A=\int_{0}^{\infty} \alpha \varepsilon C e^{-\alpha u} \cos \frac{2 \pi u}{\tau} d u=\varepsilon C \frac{\alpha^{2} \tau^{2}}{4 \pi^{2}+\alpha^{2} t^{2}}, \\
& B=\int_{0}^{\infty} \alpha \varepsilon C e^{-\alpha u} \sin \frac{2 \pi u}{t} d u=\varepsilon C^{\prime} \frac{2 \pi \alpha \tau}{4 \pi^{2}+\alpha^{2} \pi^{2}} .
\end{aligned}
$$

Somit - unter weiterer Anwendung der auf p. $723 \mathrm{ab}-$ geleiteten Formeln - für die scheinbare Kapazität des Kondensators:

$$
C^{\prime}=C\left[1+\varepsilon \cdot \frac{\alpha^{2} \tau^{2}}{4 \pi^{2}+\alpha^{2} \tau^{2}}\right]
$$

also mit zunehmender Periodendauer steigende Kapazität, und für den reziproken Wert des scheinbaren Widerstandes:

$$
L^{\prime}=L+\varepsilon C-\pi_{4}^{4} \pi^{2}+\alpha^{2} \tau^{2},
$$

also Zunahme des scheinbaren Leitvermögens und damit der in Wärme umgewandelten Energie mit abnehmender Periodendauer.

Die qualitative Übereinstimmung mit den empirischen Resultaten ist wieder vorhanden.

$b_{3}$ ) Modifikation der Pellatschen Theorie.

Eine Erweiterung der Pellatschen Theorie erhält man durch folgende Annahmen:

Die dielektrische Verschiebung besteht aus einem Bestandteil (normale Verschiebung), der jeweils der momentanen Feldintensität proportional ist, und einer Summe von Gliedern, deren jedes für sich nach einem analogen Gesetz, wie es die Pellatsche Theorie annimmt, einem Grenzwert zustrebt, bei denen aber die einer bestimmten Feldintensität entsprechenden Endbeträge und die Zeitkonstanten verschieden sind. Also:

$$
\mathfrak{D}=K\left(\mathbb{E}+\Sigma \mathfrak{D}_{i}^{\prime},\right.
$$


wobei $\mathscr{D}_{i}^{\prime}$ der Differentialgleichung genügt:

$$
\frac{d \mathfrak{D}_{i}^{\prime}}{d t}=\alpha_{i}\left[\varepsilon_{i} K\left(5-\mathfrak{D}_{i}^{\prime}\right] .\right.
$$

Im stationären Endzustande bei $\mathbb{E}=\mathbb{E}_{0}$ und $t=\infty$ wird:

$$
D_{\infty}=E_{0} K\left\{1+\Sigma \varepsilon_{i}\right\} \text {. }
$$

Wird ein Kondensator zur Zeit $t=0$ plötzlich geladen, so ist der Rückstandsstrom gegeben durch:

$$
\begin{gathered}
\mathfrak{D}_{t}=K \mathbb{E}_{0}\left\{1+\sum \varepsilon_{i}\left(1-e^{-a_{i} t}\right\},\right. \\
\left.i=-\frac{1}{4 \pi} d \mathscr{D}_{t}=\frac{K\left(\mathfrak{E}_{0}\right.}{4 \pi} \sum \alpha_{i} \varepsilon_{i} e^{-a_{i} t}\right) .
\end{gathered}
$$

Die Zeitfunktion $f(t)$, die den Abfall des Rückstandsstromes darstellt, ist also durch eine Summe von einfachen Exponentialfunktionen gegeben. Da jede Funktion, die stetig abnimmt und deren sämtliche Ableitungen ebenfalls stetig abnehmen, mit beliebiger Annäherung durch eine solche Summe von Exponentialfunktionen darstellbar ist, genügt die modifizierte Theorie jeder empirisch gefundenen Form für $f(t)$, welche obiger Bedingung bezüglich der Derivierten entspricht.

Die Gesetze der Superposition bei beliebig veränderlicher Feldintensität $\mathbb{E}_{t}$ gelten natürlich für die Summe der Exponentialfunktionen ebenso wie für die einzelne im früheren Falle der Pellatschen Theorie. Speziell für einfach periodische Wechselfelder ergibt sich:

$$
\begin{aligned}
\mathfrak{D} & =\sin \frac{2 \pi t}{\tau} \cdot K \xi_{0}\left\{1+\sum \varepsilon_{i} \frac{\alpha_{i} \tau^{2}}{4 \pi^{2}+\alpha_{i}{ }^{2} \tau^{2}}\right\}, \\
& -\cos \frac{2 \pi t}{\tau} \cdot K \xi_{0} \sum \varepsilon_{i} \frac{2 \pi \alpha_{i} \tau}{4 \pi^{2}+\alpha_{i}{ }^{2} \tau^{2}}
\end{aligned}
$$

und analog wie früher für die scheinbare Kapazität und die scheinbare Leitfähigkeit Ausdrücke, die nur durch die Einfügung des Summenzeichens modifiziert sind.

Diese Formeln lassen sich nun noch in anderer Weise darstellen. Statt einer endlichen Anzahl von Gliedern der Form $\mathfrak{D}_{i}=\varepsilon_{i} K \&$ kann man eine unendliche Anzahl annehmen, deren Zeitkonstanten $\alpha_{i}$ kontinuierlich zwischen den Werten 0 und $\infty$ abgestuft sind. An Stelle der Sättigungswerte $\varepsilon_{i} K E$ tritt dann eine Funktion $K \Subset \varepsilon(\alpha) d \alpha$, die angibt, welcher Betrag rom gesamten Verschiebungsvektor auf jene Bestandteile entfällt, deren 
Zeitkonstanten zwischen den Grenzen $\alpha$ und $\alpha+d \alpha$ liegen, analog wie man z. B. in der Theorie der Strahlung die Intensitätsverteilung über verschiedene Wellenlängen $\lambda$ darstellt durch eine Funktion $f(\lambda)$ und die Gesamtintensität setzt:

$$
r=\int_{0}^{\infty} f(\lambda) d \lambda .
$$

Die obigen Gleichungen nehmen dann folgende Form an: Im stationären Zustande für $t=\infty$ bei konstanter Feldstärke $\mathfrak{E}_{0}$ :

$$
\mathfrak{D}_{\infty}=K \mathscr{E}_{0}\left\{1+\int_{0}^{\infty} \varepsilon(\alpha) d \alpha\right\} ;
$$

bei plötzlicher Einschaltung eines Feldes $E_{0}$ zur Zeit $t=0$ :

$$
\begin{aligned}
\mathfrak{D}_{t} & =K \mathfrak{S}_{\mathrm{c}}\left\{1+\int_{0}^{\infty} \varepsilon(\alpha)\left[1-e^{-a t}\right] d \alpha\right\}, \\
i & =-\frac{1}{4 \pi} \cdot \frac{d \mathfrak{I}_{t}}{d t}=f(t)=\frac{K}{4 \pi} \mathfrak{E}_{0} \int_{0}^{\infty} \alpha \cdot \varepsilon(\alpha) e^{-\alpha t} d \alpha ;
\end{aligned}
$$

bei einfach periodischem Felde $\mathbb{E}=\mathbb{E}_{0} \sin 2 \pi t / \tau$ :

$$
\begin{aligned}
\mathscr{D} & =\sin \frac{2 \pi t}{\tau} \cdot K\left(\Xi_{0}\left\{1+\int_{0}^{\infty} \frac{\varepsilon(\alpha) \alpha^{2} \tau^{2}}{4 \pi^{2}+\alpha^{2} \tau^{2}} d \alpha\right\},\right. \\
& -\cos \frac{2 \pi t}{\tau} \cdot K\left(\Xi_{0} \int_{0}^{\infty} \frac{2 \pi \varepsilon(\alpha) \alpha \tau}{4 \pi^{2}+\alpha^{2} \frac{\tau}{\tau^{2}}} d \alpha .\right.
\end{aligned}
$$

Man kann nun auch umgekehrt die Forderung stellen, die Funktion $\varepsilon(\alpha)$ zu bestimmen, wenn z. B. der zeitliche Verlauf des Rückstandsstromes $i=f(t)$ empirisch ermittelt ist.

Es wurde bereits erwähnt, daB empirisch mit groBer Annäherung sich die Formel $i=f(t)=b \mathfrak{E}_{0}, t^{-n}$ ergibt.

Aus der Gleichung:

$$
\frac{K^{r}}{4 \pi} \int_{0}^{\infty} \alpha \cdot \varepsilon(\alpha) e^{-\alpha t} d \alpha=b \cdot t^{-n}
$$


erhält man dann $\left.{ }^{1}\right):$

oder

$$
\epsilon \cdot \varepsilon(\alpha)=\frac{4 \pi b}{K} \cdot \frac{1 \cdot}{T(1-n)} \cdot \alpha^{-(1-n)}
$$

$$
\varepsilon(\alpha)=\frac{4 \pi b}{K} \cdot \frac{1}{T(1-n)} \cdot \alpha^{-(2-n)} .
$$

Da $n<1$, also $(2-n)$ positiv ist, wird für $\alpha=0$ die Funktion $\varepsilon(\alpha)=\infty$; auch müBte im stationären Zustande die durch

$$
\mathfrak{D}_{\infty}=K \mathfrak{E}_{0}\left(1+\int_{0}^{\infty} \varepsilon(\alpha) d \omega\right)
$$

gegebene Verschiebung unendlich gro $B$ werden, und zwar durch die Integration von Null bis zu einem beliebig kleinen $\alpha_{1}$, während $\int_{a_{3}}^{\infty}$ endlich bleibt. Es wurde bereits im Teile I, Abschnitt 1, p. 715, erwähnt, daß die Annahme exakter Gültigkeit der empirischen Näherungsformel $i=b \cdot t^{-n}$ zu unwahrscheinlichen Konsequenzen führt. Nimmt man an, daß für sehr große Zeiten diese Formel nicht mehr gilt, sondern daB dann die wirkliche Stromstärke rascher absinkt und $\mathrm{daB} \int_{0}^{\infty} i d t$ endlich bleibt, so ist dann auch die Funktion $\varepsilon(\alpha)$ zu modifizieren, und zwar gerade in jenem Gebiet, für welches $\alpha=0$ oder sehr klein ist; denn der Rückstandsstrom nach sehr langer Zeit ( $t$ groB) ist bedingt durch jene Bestandteile der viskosen Verschiebung, die sich sehr langsam ändern ( $\alpha$ sehr klein). Es genügt also im Intervalle von $\alpha=0$ bis $\alpha=\alpha_{1}$, wo $\alpha_{1}$ sehr klein sein kann, $\varepsilon(\alpha)$ durch eine andere Funktion als die oben angegebene zu ersetzen, die der Bedingung genügt: $\int_{0}^{a_{1}} \varepsilon(\alpha) d c$ $=$ endlich, für $\alpha<\alpha_{1}$ aber die obige Form beizubehalten; das Resultat ist, $\mathrm{daB} \int_{0}^{\infty} \varepsilon(\alpha) d \alpha$ endlich bleibt und daB die Stromstärke $i=\int_{0}^{\infty} \alpha \varepsilon(\alpha) e^{-\alpha t} d \alpha$ bis zu groBen Werten von $t$ durch die Formel $i=b . t^{-n}$ dargestellt wird, erst dann rascher als nach dieser Formel gegen Null konvergiert und daB die „Rückstandsladung" $\int_{0}^{\infty} i d t$ endlich bleibt. Für die Vorgänge, die

1) Vgl. Serret, Differential- und Integralrechnung, III/1, p. 190. 
sich im periodisch wechselnden Felde abspielen, ist die Form der Funktion $\varepsilon(\alpha)$ für kleine Werte des Argumentes unwesentlich, solange die Periodendauer $\tau$ nicht sehr groB wird, da die dabei auftretenden Koeffizienten $A$ und $B$ durch Integration von Produkten $\alpha \varepsilon(\alpha)$, bzw. $\alpha^{2} \varepsilon(\alpha)$ entstehen, somit die Integration im Intervall von 0 bis $\alpha_{1}$ ( $\alpha_{1}$ sehr klein) Beträge liefert, die unendlich klein von der ersten, bzw. zweiten Ordnung sind.

Die Charakterisierung eines Dielektrikums erfolgt also nach der modifizierten Pellatschen Theorie folgendermaBen:

Außer der Dielektrizitätskonstante $K$ und dem spezifischen Leitvermögen $\lambda$ des Mediums ist noch eine Funktion $\varepsilon(\alpha)$ im Bereich $\alpha=0$ bis $\alpha=\infty$ anzugeben; das Integral

$$
\int_{0}^{\infty} \alpha \cdot \varepsilon(\alpha) e^{-\alpha t} d \alpha=f(t)
$$

liefert dann das Gesetz des zeitlichen Verlaufes des Rückstandsstromes; umgekehrt kann die Funktion $\varepsilon(\alpha)$ aus der empirisch gefundenen Funktion $f(t)$ ermittelt werden. Vermöge der Gültigkeit des Superpositionsprinzipes, die sich aus den Annahmen der Theorie ergibt, lassen sich dann die Gesetze des Stromverlaufes bei beliebig variierender Feldintensität prinzipiell ableiten, also insbesondere die Vorgänge im Wechselfelde: die scheinbare Kapazitätsänderung bei Variation der Periode und die in Wärme umgewandelte Energie, ferner die damit zusammenhängenden ponderomotorischen Kräfte, die ein Dielektrikum bei relativer Rotation zu einem konstanten elektrischen Felde erfährt.

Die Fassung dieser erweiterten Theorie ist also allgemein genug, um beliebige empirisch gefundene oder noch zu findende Formen des zeitlichen Verlaufes der Rückstandsbildung darstellen zu können und präzisiert genug, um die quantitative, nicht bloB qualitative Ableitung der übrigen Hauptformen anomalen Verhaltens aus der Rückstandsbildung zu ermöglichen.

$\mathrm{BloB}$ in einem Detail ergibt sich ein Widerspruch zwischen den Resultaten der theoretischen Ableitung und dem direkten Ergebnis des Experimentes; die im Wechselfelde umgewandelte Energie und damit die Drehungsmomente im rotierenden Felde 
müssen nach der Theorie proportional dem Quadrat der Feldintensität sein, während von einigen Beobachtern eine andere Beziehung (proportional $\mathfrak{C}^{n}$, wo $n=1,5$ bis 1,96 , vgl. p. 720 ) gefunden wurde. In diesen Fällen spielt vielleicht die anomale Art der Leitung mit, deren Effekte sich über die der dielektrischen Nachwirkung überlagern.

\section{$b_{4}$ ) Molekulartheoretische Bedeutung der modifizierten Pellatschen Theorie.}

Die Zerlegung des tatsächlich vorhandenen Verschiebungsvektors $\mathfrak{D}$ in eine beliebig große Anzahl von Teilbeträgen, die alle einer bestimmten Differentialgleichung genügen, aber für die darin enthaltenen Parameter $\alpha$ und $\varepsilon$ verschiedene Werte aufweisen, erscheint auf den ersten Blick als eine gekünstelte, rein mathematische Fiktion, die schließlich nur darauf hinausläuft, für die Darstellung einer komplizierten empirisch gegebenen Funktion beliebig viele Konstanten einzuführen. Es soll nun gezeigt werden, wie diese Fiktion einer einfachen physikalischen Interpretation fähig ist.

In der vormaxwellschen Zeit wurden die dielektrischen Erscheinungen gedeutet als bedingt durch die Einlagerung kleiner (molekularer) leitender Teilchen; diese Anschauung gipfelt in der bekannten Clausius-Mosottischen Theorie.

Eine Zeitlang wurden durch die Maxwellsche Formulierung der Grundgleichungen die molekularphysikalischen Betrachtungsweisen auf dem Gebiete der Dielektrika ganz zurückgedrängt.

Die Anschauungen der modernen Ionen- und Elektronentheorie führten aber solche wieder ein, und zwar konnten die Vorstellungen der alten Theorie mit leichten Modifikationen wieder verwendet werden. An Stelle des leitenden Partikels oder Moleküls - grob versinnlicht durch eine kleine Metallkugel in einem isolierenden Medium eingebettet - tritt das aus entgegengesetzt geladenen Ionen (negatives Elektron und positives Restatom) bestehende Molekül. Die relative Verschiebbarkeit der beiden Bestandteile ersetzt die Leitung im alten Sinne. Die Moleküle als Ionenkomplexe können nun auch als "Resonatoren" aufgefaßt werden, indem die aus der Gleichgewichtslage gebrachten Ionen eine bestimmte Dauer der 
Eigenschwingung besitzen. Die Erscheinungen der normalen und anomalen Dispersion elektrischer Wellen, sowie die damit zusammenhängende Absorption werden in der modernen Theorie von diesem Gesichtspunkte aus behandelt.

Es liegt nun nahe anzunehmen, daß neben Molekülen, deren Ionen eine Eigenschwingung von bestimmter Dauer und bestimmtem Dämpfungsverhältnis bedingen, auch solche vorhanden seien, bei denen die Dämpfung so gro $B$ ist, da $B$ an Stelle einer Schwingung eine aperiodisch gedämpfte Bewegung auftritt. Unter dem EinfluB eines plötzlich auftretenden, dann konstant bleibenden elektrischen Feldes stellt sich dann der neue Gleichgewichtszustand derart her, daB die Abweichung von diesem nach einer Exponentialfunktion $e^{-\alpha t}$ abnimmt.

Die molekularphysikalische Deutung der Pellatschen Theorie wäre daher folgende: Neben den Molekülen, die als Resonatoren mit bestimmter (sehr kleiner) Schwingungsdauer einem relativ langsam veränderlichen elektrischen Felde ohne merkliche Phasendifferenz folgen, sind in einem anomalen Dielektrikum auch Moleküle vorhanden, in denen die Verschiebung der Ionen aperiodisch gedämpft erfolgt, und zwar so, daB die Konstante $\alpha$ der obigen Formel für alle Moleküle den gleichen Wert besitzt. Die Größe $\varepsilon$ gibt an, in welchem Verhältnis der VerschiebungslluB dieser aperiodisch gedämpften Moleküle zu dem der oszillatorisch beweglichen steht.

In der modifizierten Theorie wird angenommen, daB nicht eine Gattung solcher aperiodisch gedämpfter Ionenkomplexe mit bestimmter Zeitkonstante $\alpha$ vorhanden sei, sondern eine große Anzahl verschiedener Gattungen mit verschiedenen Werten ihrer Zeitkonstanten $\alpha_{i}$, die in verschiedener Anzahl (proportional $\varepsilon_{i}$ ) pro Volumeinheit vorhanden sind.

Eventuell kann man die möglichen Werte der Dämpfung als kontinuierlich abgestuft auffassen und die Funktion $\varepsilon(\alpha)$ gibt an, nach welchem Gesetze die Häufigkeit der von 0 bis $\infty$ variierenden $\alpha$-Werte verteilt ist.

Die Zerlegung einer empirisch gegebenen Funktion in eine Summe einfacher Exponentialfunktionen entspricht daher physikalisch einer Sonderung der Wirkungen, die von verschiedenen Gruppen unter sich gleichartiger Moleküle hervorgebracht werden. 


\section{Dritter Teil.}

Experimentelle Beiträge zur Untersuchung der Rückstandsbildung.

1. Versuchsanordnung.

Um den Rückstandsstrom bezüglich seines zeitlichen Verlaufes, seiner Abhängigkeit von Ladungsdauer, elektromotorischer Kraft, Temperatur usw. zu untersuchen, wurde die Methode der direkten galvanometrischen Messung angewendet. Das benutzte Instrument war ein hoch empfindliches Drehspulengalvanometer, dessen Reduktionsfaktor $3,19 \cdot 10^{-10} \frac{A}{\text { pars }}$ betrug; hieraus und aus der Schwingungsdauer und Dämpfung der Spule berechnete sich der ballistische Reduktionsfaktor zu $1,34 \cdot 10^{-9} \frac{C b}{\text { pars }}$.

Nebenstehende Fig. 4 zeigt die Schaltung an; der zu untersuchende Kondensator $K$ ist einerseits mit einem Punkt von konstantem Potential $+E$ verbunden, andererseits über das Galvanometer $G$ zur Erde abgeleitet. Um den heftigen Ausschlag des Galvanometers beim Ein- oder Ausschalten der elektromotorischen Kraft $E$ zu vermeiden, ist ein Schlüssel $S_{\mathrm{I}}$ als Kurzschlub der Galvanometerleitung angebracht; zugleich gestattete es dieser Schlüssel in Verbindung mit dem Schlüssel $S_{\text {II }}$ jederzeit, ohne Unterbrechung des den Kondensator durch-

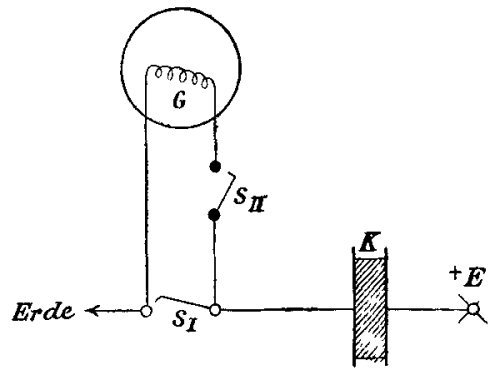

Fig. 4. fließenden Stromes, den Nullpunkt zu prüfen. Bei Anwendung kleiner Werte von $E$ konnte bei geöffnetem Schlüssel $S_{\mathrm{I}}$ aus dem ballistischen Ausschlage die Kapazität des Kondensators $K$ bestimmt werden.

$\mathrm{Da}$ in verschiedenen Versuchsreihen sehr verschiedene Meßbereiche nötig waren, wurden diese nach Bedarf geändert. Eine Erhöhung des MeBbereiches (Erniedrigung der Empfindlichkeit) auf das Zehn-, Hundert- oder Tausendfache des normalen Wertes wurde in der üblichen Weise durch Anlegen 
von Shunts zur Galvanometerspule bewerkstelligt. Um den Meßbereich auch auf schwache Ströme (von etwa $10^{-9} \mathrm{Amp}$. abwärts bis $10^{-11} \mathrm{Amp}$.), die einen für genauere Messung zu geringen Ausschlag erzeugten, auszudehnen, wurde folgende Versuchsanordnung getroffen: Die über das Galvanometer zur Erde führende Stromleitung kann durch einen Schlüssel $S_{\text {III }}$ unterbrochen werden. Parallel zu dieser Leitung ist eine zweite geschaltet, die einen Glimmerkondensator $G K$ von 1 Mikrof. Kapazität enthält. Der Glimmerkondensator nimmt dann während der Unterbrechungsdauer $T$ infolge des (als konstant vorausgesetzten) Stromes $i$ die Ladung $i T$ an, welche durch

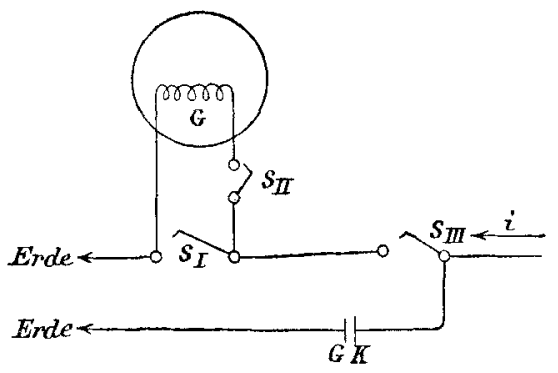

Fig. 5.

Schließen von $S_{\text {III }}$ aus dem ballistischen Ausschlag ermittelt werden kann. Ein Strom, der einen Dauerausschlag von 1 pars bewirkt, gibt pro Sekunde der Unterbrechungszeit einen ballistischen Ausschlag von 1/4,21 partes, also bei 500 Sek. Unterbrechungszeit einen Ausschlag von 119 partes, d. i. eine Erhöhung der Empfindlichkeit auf das rund 120 fache der normalen. Infolge der groBen Kapazität des Glimmerkondensators bleibt das Potential der aufgeladenen Belegung auch nach längerer Zeit klein, z. B. 0,16 Volt nach 500 Sek. im früheren Beispiel, so daB die den Strom $i$ unterhaltende elektromotorische $\operatorname{Kraft} E$ (gewöhnlich 100-300 Volt) praktisch als konstant betrachtet werden kann. Der Ladungsverlust durch Leitung und Rückstandsbildung im Glimmerkondensator selbst war zu vernachlässigen.

Unmittelbar nach der Ein- oder Ausschaltung der elektromotorischen Kraft ist die zeitliche Änderung des Rückstandsstromes eine so rapide, daß das infolge seiner starken Dämpfung 
träge Galvanometer eine Messung der zu einer bestimmten Zeit vorhandenen Stromstärke durch den gleichzeitig beobachteten Ausschlag unmöglich macht; erst nach etwa einer halben Minute kann der - nun langsamer abnehmende Ausschlag als $\mathrm{MaB}$ des Stromes angesehen werden. Um nun auch kurz nach StromschluB wenigstens einen Wert der Stromstärke zu bestimmen, wurde folgende Schaltung angewendet:

Parallel dem Galvanometer liegt eine Leitung, die den Schlüssel $S_{1}$ und einen Widerstand $W_{1}\left(W_{1}=1 \mathrm{Ohm}\right)$ enthält. An den Enden des Widerstandes $W_{1}$ liegt wieder eine Leitung, die einen Akkumulator $A$ und einen Regulierwiderstand $R$

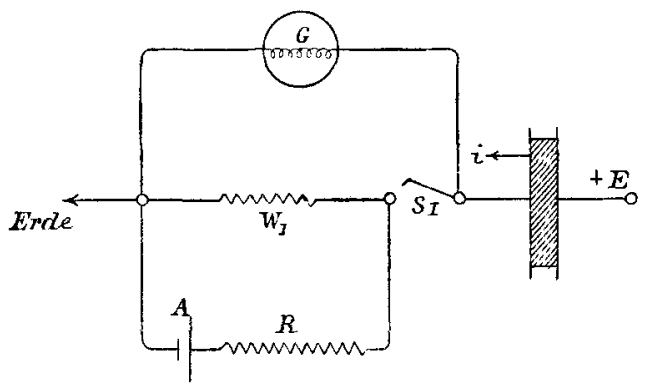

Fig. 6.

enthält. Das Verfahren ist folgendes: Durch einen Vorversuch wird auf dem Wege der Extrapolation ungefähr ermittelt, welche Stromstärke zu einer bestimmten Zeit $t_{1}$ nach Einschaltung der elektromotorischen Kraft im Galvanometer zu erwarten ist. Beim zweiten definitiven Versuche bleibt $S_{\mathrm{I}}$ zunächst geschlossen; der Akkumulator $A$ entsendet einen Zweigstrom (Hilfsstrom) in das Galvanometer, der durch Variation des Regulierwiderstandes $R$ auf eine beliebige GröBe gebracht werden kann und einen konstanten Ausschlag $a$ hervorruft. Nun wird zur Zeit $t=0$ im Hauptkreis die elektromotorische Kraft $E$ eingeschaltet; da $S_{\mathrm{r}}$ geschlossen ist und $W_{1}(1 \mathrm{Ohm})$ gegenüber dem Widerstande der Galvanometerspule $(10000 \mathrm{Ohm}$ ) immer noch als KurzschluB aufgefaBt werden kann, geht der normale Ladungsstrom und der mit hohen An. fangswerten einsetzende Rückstandsstrom $i$ zunächst über $S_{\mathrm{I}}$ 
und $W_{1}$ zur Erde, während im Galvanometer der Hilfsstrom den konstanten Ausschlag $a$ erhält. Zu jener Zeit $t=t_{1}$, für welche auf Grund des Vorversuches zu erwarten ist, daB $i=a$ ist, wird der Schlüssel $S_{\mathrm{r}}$ geöffnet. Es wird damit gleichzeitig der Hilfsstrom $a$ unterbrochen und der zu messende Strom $i$ über das Galvanometer geleitet. War wirklich im Momente der Unterbrechung $i=a$, so bleibt in diesem Momente die abgelenkte Galvanometerspule im Gleichgewichte, um dann der Abnahme von $i$ entsprechend ihren Ausschlag zu verringern; war aber im Momente der Unterbrechung $i$ größer oder kleiner als $a$, so erfolgt auch eine stoBartige VergröBerung oder Verkleinerung des Ausschlages. Auf diese Weise kann also die Stromstärke $i$ bestimmt werden für eine relativ kurze Zeit (in praxi etwa 10 Sek.) nach StromschluB.

\section{Versuchsresultate.}

Zunächst soll in drei Beispielen (Versuchsreihen 1, 2 und 3) der auf p. 714 besprochene Unterschied der beiden Typen anomalen Ladungsstromes gezeigt werden.

Versuchsreihe 1.

Zyjinderkondensator mit Petroleum gefüllt; $t$ Zeit in Sekunden von StromschluB an gerechnet, $E$ Potentialdifferenz in Volt, $i$ beobachteter Strom in Skalenteilen, $a$ stationärer Endwert desselben, $y$ gegeben durch $i-a$.

Von $t=0$ bis $t=1000: E=300$ Volt; für $t>1000: E=0$.

\begin{tabular}{r|r|r||r|r|r}
\hline$t$ & $i$ & $y$ & $t$ & \multicolumn{1}{c||}{$i$} & $y$ \\
\hline \hline 0 & - & - & 180 & 126,3 & 0,8 \\
15 & 240 & 114,5 & 240 & 126 & 0,5 \\
30 & 188 & 62,5 & 300 & 126,2 & 0,7 \\
45 & 168 & 42,5 & 660 & 125,5 & 0,0 \\
60 & 151 & 25,5 & 900 & 125,5 & 0,0 \\
90 & 134 & 8,5 & 1000 & - & - \\
120 & 129 & 3,5 & 1020 & $-0,2$ & $-0,2$ \\
150 & 127 & 1,5 & 1060 & $-0,1$ & $-0,1$
\end{tabular}


Versuchsreihe 2.

Plattenkondensator mit Toluol gefüllt; Bezeichnung wie bei 1 . $t=0$ bis $t=3600 \mathrm{sec}: E=200$ Volt; $t>3600$ sec: $E=0$ Volt.

\begin{tabular}{r|c|c||c|c|c}
\hline \multicolumn{1}{c|}{$t$} & $i$ & $y$ & $t$ & $i$ & $y$ \\
\hline 0 & - & - & 300 & 110 & 32 \\
30 & 375 & 297 & 360 & 105 & 27 \\
60 & 260 & 182 & 420 & 101 & 23 \\
90 & 182 & 104 & 480 & 99 & 21 \\
120 & 155 & 77 & 540 & 95 & 17 \\
150 & 140 & 62 & 600 & 94 & 16 \\
180 & 130 & 52 & 3600 & 78 & 0,0 \\
240 & 117 & 39 & 3650 & $-0,5$ & 0,5
\end{tabular}

Versuchsreibe 3.

Glaskondensator (Eprouvette, als Belegungen innen und außen Schwefelsäure); Bezeichnung wie früher.

Von $t=0$ bis $t=900 \mathrm{sec}: E=300$ Volt; für $t>900 \mathrm{sec}: E=0$ Volt.

\begin{tabular}{r|c|c||c|c}
\hline \multicolumn{1}{c|}{$t$} & $i_{1}$ & $y_{1}=i_{1}-a$ & $t$ & $i_{2}=y_{2}$ \\
\hline \hline 0 & - & - & 900 & - \\
10 & 22 & 16 & 910 & -18 \\
20 & 16 & 10 & 920 & $-9,8$ \\
30 & 12,6 & 6,6 & 930 & $-7,3$ \\
50 & 11,1 & 5,1 & 950 & $-5,0$ \\
00 & 9,1 & 3,1 & 980 & $-3,9$ \\
100 & 9,0 & 3,0 & 1000 & $-3,1$ \\
150 & 8,0 & 2,0 & 1050 & $-2,0$ \\
600 & 6,0 & 0,0 & 1500 & $-0,0$ \\
890 & 6,0 & 0,0 & 1800 & $-0,0$
\end{tabular}

Das Resultat ist also, daß in den Versuchsreihen 1 und 2 der Strom allmählich auf einen stationären Endwert abnimmt, nach Ausschaltung der elektromotorischen Kraft aber ein Strom merklicher Intensität überhaupt nicht vorhanden ist, daB dagegen in der Versuchsreihe 3 zunächst analog wie in den ersten beiden Fällen eine Abnahme des Stromes erfolgt, nach Ausschaltung der elektromotorischen Kraft aber ein entgegen- 
gesetzt gerichteter Strom auftritt, dessen Stärke innerhalb der Beobachtangsfehler der GröBe $y_{1}$, d. i. dem Überschusse des Stromes $i_{1}$ über seinen stationären Endwert, gleich ist.

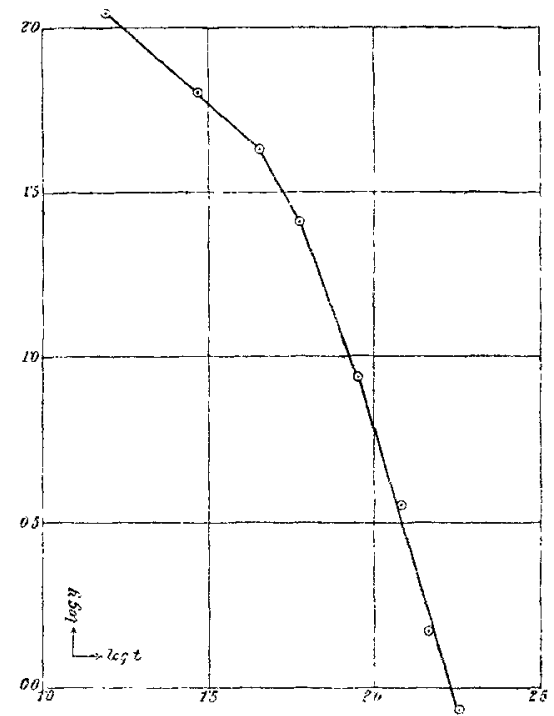

Fig. 7 zu Versuchsreihe 1, Petroleumkondensator.

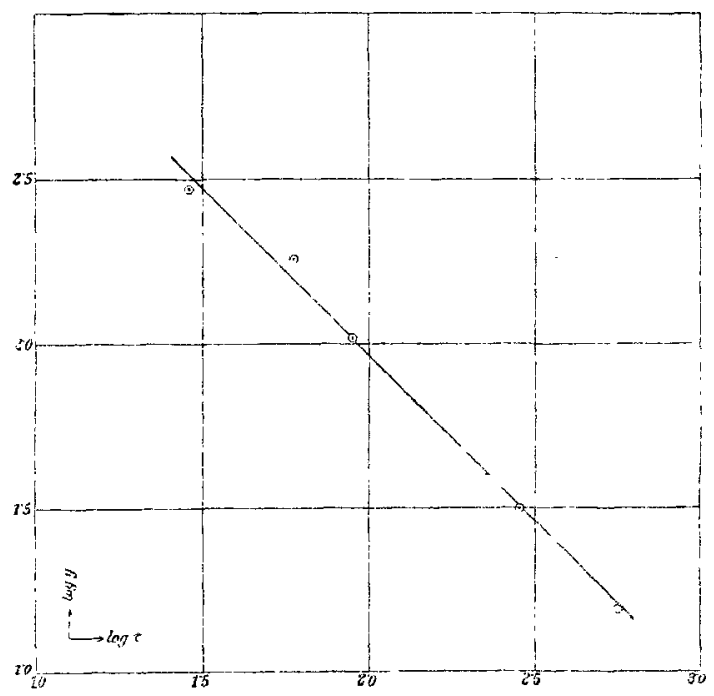

Fig. 8 zu Versuchsreibe 2, Toluolkondensator. 
Die Kurven, Figg. 7, 8, und 9 geben ein Bild von den Gesetzen des zeitlichen Verlaufes der variablen Bestandteile

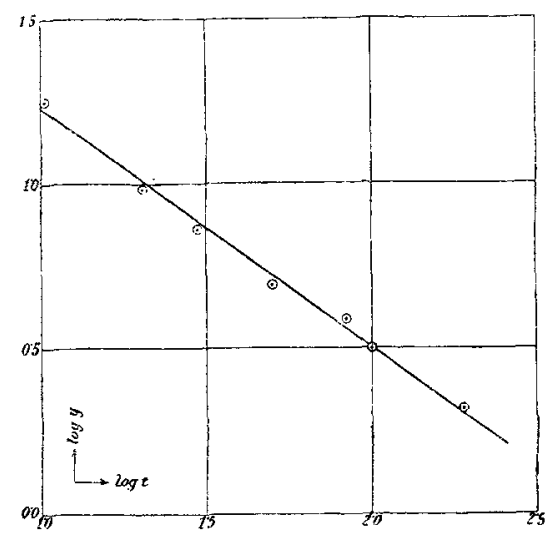

Fig. 9 zu Versuchsreihe 3, Glaskondensator.

$y=i-a$; die Abszissen stellen $\log t$, die Ordinaten $\log y$ dar; für Figg. 8 und 9 erhält man angenähert Gerade: also da

daraus

$$
\log y=\text { Const. }-n \log t
$$

$$
y=B \cdot t^{n} \text {. }
$$

Für Fig. 7 dagegen tritt an Stelle einer Geraden eine stark nach abwärts gekrümmte Kurve, die allerdings dann in eine Gerade überzugehen scheint.

Trotz der mehr oder weniger großen Analogie in der Form des zeitlichen Ganges sind die Fälle 1 und 2 scharf zu sondern von 3. Bei 1 und 2 liegt ein nicht reversibler anomaler Ladungsstrom vor, der theoretisch auf die Eigenschaften eines Mediums mit Ionenleitung zurückgeführt werden kann. Bei 3 liegt ein reversibler anomaler Strom, also eigentliche Rückstandsbildung vor. Bei flüssigen Dielektrikas ist vom Verfasser immer nur der erste Typus konstatiert worden, bei festen Dielektrikas der zweite.

Die von vielen Beobachtern (vgl. p. 716) gefundene und durch den oben angeführten Versuch 3 bestätigte Formel:

$$
i_{1}-a=-i_{2}=B \cdot t^{-n}
$$

für den zeitlichen Verlauf der Rückstandsströme wurde bisher 
immer nur für relativ kleine Zeitintervalle (bis zu einer Stunde etwa) experimentell geprüft.

Die beiden folgenden Versuchsreihen erstrecken sich über weit länģere Zeiten.

\section{Versuchsreihe 4.}

Glimmerkondensator von 1 Mikrofarad Kapazität, durch 69 Stunden $=248400$ Sek. auf 300 Volt geladen; zur Zeit $t=0$ entladen; für den Entladungsstrom $i_{2}$ wurde gefunden:

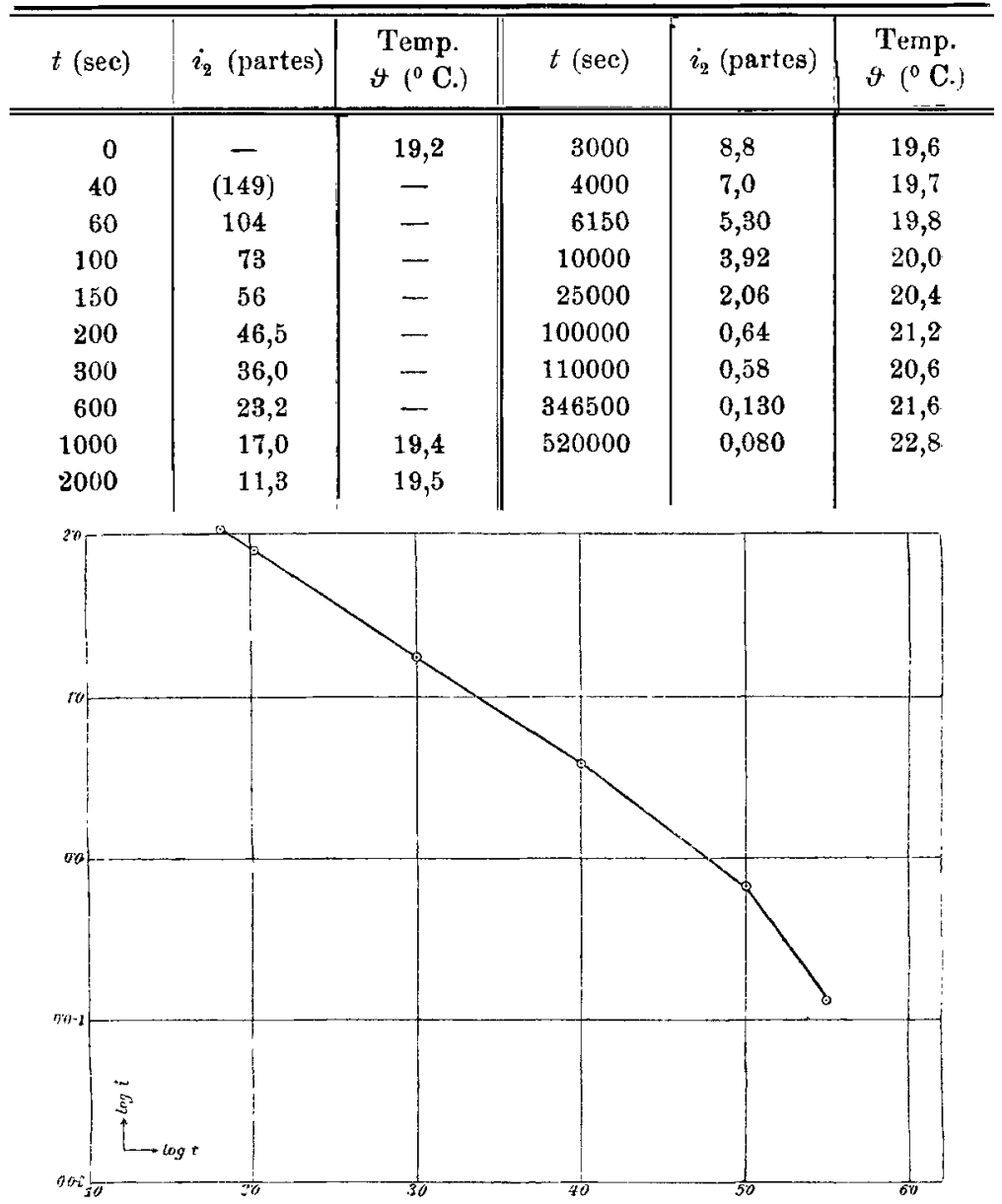

Fig. 10 zu Versuchsreihe 4, Glimmerkondensator. 
Die graphische Darstellung gibt bis zu ca. $t=10000 \mathrm{sec}$ $(\log t=4,0)$ einen linearen Verlauf, also Gültigkeit der obigen Formel; für $t>100000$ fällt der Strom rascher, als es der Formel entspricht. Doch kann daraus noch nicht auf ibre Ungültigkeit für hohe Werte von $t$ geschlossen werden.

Der Voraussetzung nach sollte die Formel $i=B t^{-n}$ gelten für den anomalen Ladungsstrom sowie für den Entladungsstrom dann, wenn der Kondensator unendlich lange Zeit vorher auf konstanter Potentialdifferenz geladen gehalten wurde. Nach endlicher Ladungsdauer $\delta$ ist nach dem Superpositionsprinzipe $z u$ erwarten, da $B$ der Entladungsstrom

$$
i_{2}=f(t)-f(t+\delta)=B\left[t^{-n}-(t+\delta)^{-n}\right] .
$$

Da im vorliegenden Falle $\delta$ zwar groB (rund $250000 \mathrm{sec}$ ), aber nicht unendlich ist, so wird beim Entladungsstrom das Glied $(t+\delta)^{-n}$ für kleine Werte von $t$ zu vernachlässigen, für große Werte von $t$ aber merklich sein.

Eine Beobachtung des über den eventuellen konstanten Leitungsstrom übergelagerten Ladungsstromes, die direkt $f(t)$ gegeben hätte, mußte aus praktisch-technischen Gründen unterbleiben: die elektromotorische Kraft - durch Abzweigen vom Straßenstrom erhalten - war nicht vollkommen konstant, so dab ihren Variationen entsprechende normale Ladungs- und Entladungsströme sich über den zu beobachtenden Strom überlagerten und unregelmäBige, sehr bedeutende Schwankungen des Ausschlages hervorriefen.

Versuchsreihe 5.

Paraffinpapierkondensator von 5 Mikrof. Kapazität, durch 6 Tage und 5 Stunden $=450000$ Sek. auf 300 Volt geladen; zur Zeit $t=0$ entladen; für den Entladungsstrom $i_{3}$ wurde gefunden:

\begin{tabular}{r|c|c||c|c|c}
\hline$t$ (sec) & $i_{2}$ (partes) & $\begin{array}{c}\text { Temp. } \\
\vartheta\left({ }^{\circ} \mathrm{C} \text { ) }\right.\end{array}$ & $t$ (sec) & $i_{2}$ (partes) & $\begin{array}{c}\text { Temp. } \\
\vartheta\left({ }^{\circ} \mathrm{C} .\right)\end{array}$ \\
\hline \hline 0 & - & 20,4 & 180 & 8580 & - \\
60 & 22500 & - & 200 & 8080 & - \\
100 & 19000 & - & 300 & 6360 & - \\
120 & 11200 & - & 600 & 4550 & -
\end{tabular}




\begin{tabular}{|c|c|c|c|c|c|}
\hline$t$ (sec) & $i_{2}$ (partes) & $\begin{array}{l}\text { Temp. } \\
\vartheta\left({ }^{0} \mathrm{C}\right)\end{array}$ & $t(\mathrm{sec})$ & $i_{2}$ (partes) & $\begin{array}{l}\text { Temp. } \\
\vartheta\left({ }^{\circ} \mathrm{C} .\right)\end{array}$ \\
\hline 1000 & 3640 & - & $2,17 \cdot 10^{6}$ & 139 & 19,0 \\
\hline 1500 & 3090 & - & $2,33 \cdot 10^{6}$ & 186,5 & 22,8 \\
\hline 3000 & 2420 & 21,0 & $2,42 \cdot 10^{8}$ & 201,5 & 23,6 \\
\hline 10000 & 1640 & 20,6 & $2,51 \cdot 10^{6}$ & 213 & 24,2 \\
\hline 93000 & 748 & 19,8 & $2,68 \cdot 10^{6}$ & 202 & 24,4 \\
\hline 250000 & 596 & 22,6 & $3,11 \cdot 10^{8}$ & 202 & 26,0 \\
\hline 332000 & 491 & 21,4 & $3,19 \cdot 10^{6}$ & 209 & 27,0 \\
\hline 508500 & 403 & 22,4 & $3,72 \cdot 10^{6}$ & 170 & - \\
\hline 768600 & 287 & - & $10,89 \cdot 10^{6}$ & 17,0 & 17,2 \\
\hline $1,20 \cdot 10^{6}$ & 186,5 & 19,8 & $11,69 \cdot 10^{6}$ & 16,0 & 18,0 \\
\hline $1,48 \cdot 10^{6}$ & 154,5 & 18,4 & $12,11 \cdot 10^{6}$ & 13,1 & 14,0 \\
\hline $1,72 \cdot 10^{8}$ & 162 & 20,2 & $15,97 \cdot 10^{8}$ & 8,2 & 12,8 \\
\hline $1,80 \cdot 10^{\circ}$ & 155 & 19,7 & $15,98 \cdot 10^{8}$ & 18,0 & 18,8 \\
\hline $2,08 \cdot 10^{3}$ & 155 & 20,0 & & & \\
\hline
\end{tabular}

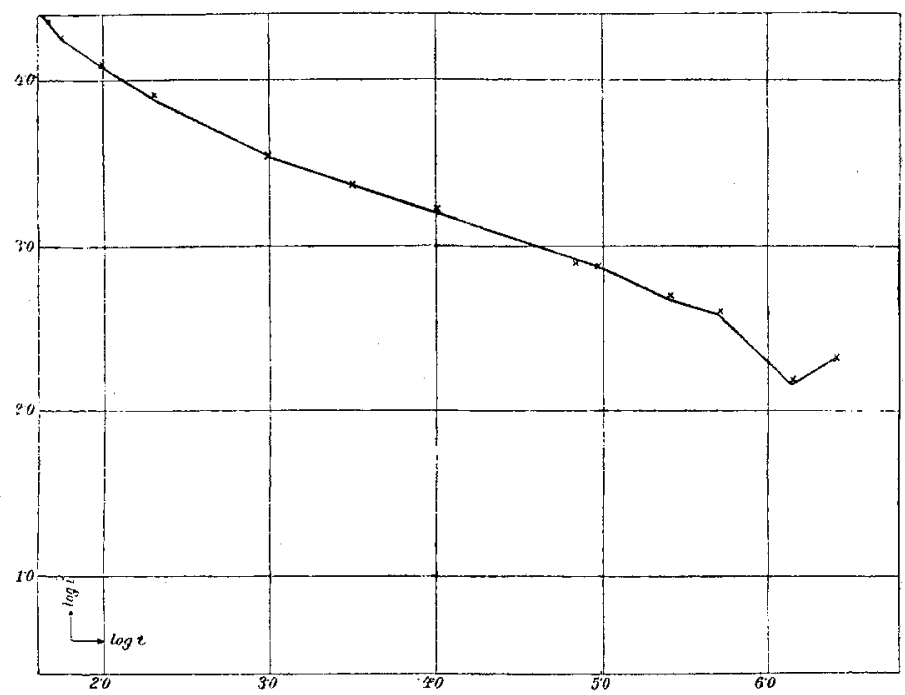

Fig. 11 zu Versuchsreihe 5, Paraffinpapierkondensator.

Wie die graphische Darstellung (Fig. 11) zeigt, ist hier die obige Formel weniger gut erfüllt; $\log i$ fällt zuerst rascher (entsprechend $n=0,86$ ), dann langsamer (entsprechend $n=0,34$ ) für grobe Werte von $t(t>100000)$ wird der Gang ganz unregelmäBig, in offenbarem Zusamenhang mit Temperatur. 
schwankungen. Erhöhter Temperatur entsprechen höhere Stromwerte. Nach mehr als einem halben Jahre $(t=16$ Millionen Sekunden) ist der Rückstandsstrom noch sehr merklich; da die zeitliche Änderung nun eine sehr langsame ist, kommen die Temperatureinflüsse rein zur Geltung. Einer Erhöhung der Temperatur von $12,8^{\circ} \mathrm{C}$. auf $18,8^{\circ} \mathrm{C}$. entspricht ein Anwachsen der Stromstärke von 8,2 auf 18,0 partes.

Im AnschluB hieran seien einige Versuche über die Beeinflussung der Rückstandsbildung in Glas durch die Temperatur mitgeteilt.

\section{Versuchsreibe 6.}

Glaskondensator, bestehend aus Kochkolben, Belegungen innen und außen Schwefelsäure; in fünf Versuchen bei verschiedenen Temperaturen wurde der Entladungsstrom gemessen, nachdem der Kondensator in allen fünf Fällen durch eine Zeit $\delta=1800 \mathrm{sec}$ auf 300 Volt geladen worden war. Die Tem. peraturen betrugen:

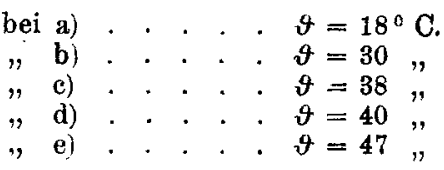

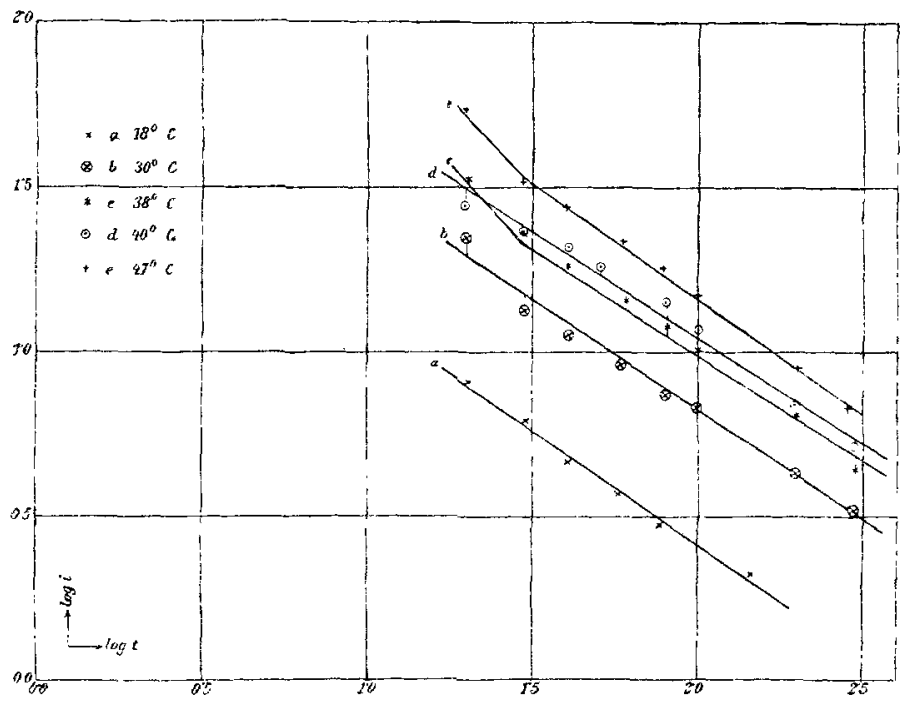

Fig. 12 zu Versuchsreihe 6, Glaskondensator. 
Unter Hinweglassung der zugehörigen Zahlentabellen sei direkt auf die graphische Darstellung in Fig. 12 verwiesen.

Es ergibt sich, daB die Kurven $\log i=f(\log t)$ angenähert Gerade von gleicher Neigung sind, aber um so höher liegen, je größer die zugeordnete Temperatur ist. Es gilt also auch angenähert die Formel $i=B \cdot t^{-n}$, und zwar ist $n$ durch die Temperatur nicht wesentlich beeinflußt, während $B$ mit steigender Temperatur zunimmt.

Mit steigender Temperatur nimmt also der Rückstandsstrom zu, ohne die Form des zeitlichen Verlaufes erheblich zu ändern.

Die folgende Versuchsreihe enthält eine Prüfung des Superpositionsprinzips. Wird ein Kondensator unter sonst gleichen Bedingungen (gleiche elektromotorische Kraft, gleiche Temperatur) bei variabler Ladungsdauer $\delta$ geladen und dann der Entladungsstrom gemessen, so sollte bei Gültigkeit des Superpositionsprinzips die Gleichung erfüllt sein:

$$
i=B t^{-n}-B(t+\delta)^{-n}
$$

Versuchsreihe 7.

Glimmerkondensator von 1 Mikrof. Kapazität; $E=300$ Volt, $\delta$ bzw. gleich 2, 10, 60, 600, 1800, 250000 Sek. Die graphische Darstellung in Fig. 13.

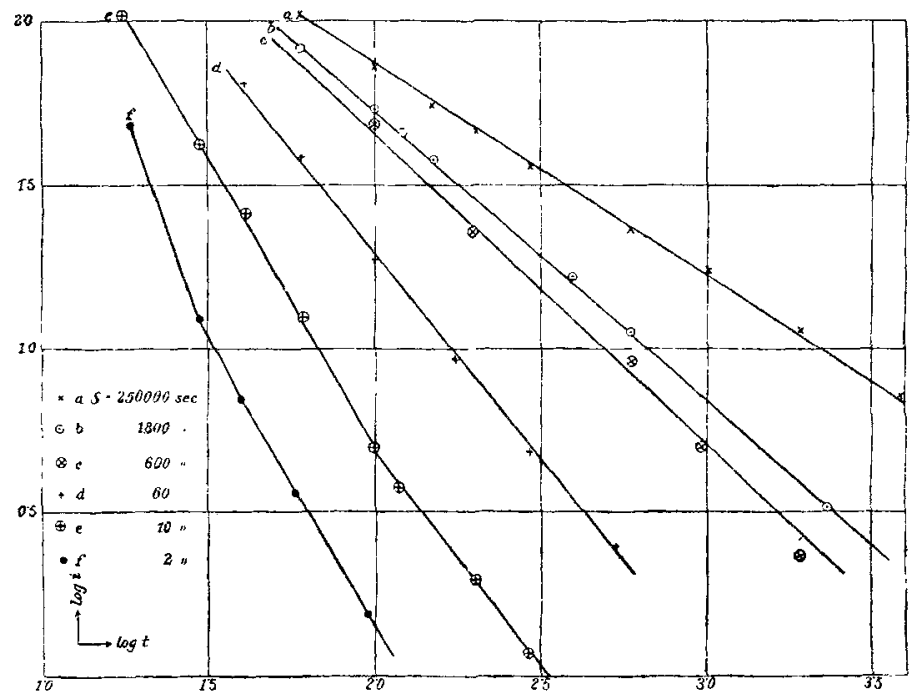

Fig. 13 zu Versuchsreihe 7, Glimmerkondensator. 
Anomalien im Verhalten der Dielektrika.

\begin{tabular}{|c|c|c|c|c|c|c|c|c|c|c|c|c|c|c|}
\hline 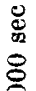 & 仓 & 1 & 1 & 1 & $\underset{\Xi}{\sigma}$ & $\ddot{\theta}$ & $\stackrel{\infty}{\Gamma}$ & 10 & $\frac{10}{0^{\prime}}$ & $\underset{\infty}{\stackrel{8}{0}}$ & $\stackrel{\infty}{\infty}$ & $\underset{\sim}{\stackrel{\theta}{\sigma}}$ & $\stackrel{-1}{=}$ & $\stackrel{\infty}{\infty}$ \\
\hline $\begin{array}{l}10 \\
11 \\
\infty\end{array}$ & $\stackrel{5}{8}$ & 8 & $\frac{\infty}{\infty}$ & $\stackrel{t a}{\rightarrow}$ & $\underset{\infty}{\rightarrow}$ & $\overrightarrow{0}$ & $\stackrel{\infty}{\infty}$ & $\begin{array}{l}\infty \\
0 \\
0\end{array}$ & $\stackrel{9}{0}$ & $\frac{\omega}{\infty}$ & $\stackrel{10}{10}$ & $\stackrel{0}{=}$ & $\underset{0}{\Xi}$ & $\infty$ \\
\hline 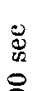 & مُهْ & $!$ & 1 & 1 & $\stackrel{8}{9}$ & $\vec{\infty}$ & as & مू & 1 & $\stackrel{8}{8}$ & $=$ & 1 & 1 & 1 \\
\hline 11 & 灾 & 8 & 兽 & $\underset{\sim}{\stackrel{0}{*}}$ & $\underset{\mathbf{N}}{\mathbf{N}}$ & $\stackrel{\infty}{\sigma}$ & $\underset{0}{\infty}$ & 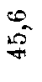 & $\stackrel{0}{\infty}$ & $\begin{array}{l}0 \\
\stackrel{0}{0}\end{array}$ & $\underset{+1}{0}$ & $\infty$ & $+\infty$ & $\stackrel{-21}{-1}$ \\
\hline 导 & $\begin{array}{l}\dot{0} \\
0 \\
0\end{array}$ & $\mid$ & 1 & 1 & 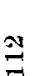 & 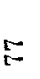 & $\underset{7}{*}$ & $\frac{n}{\infty}$ & $\underset{\infty}{\infty}$ & 1 & $\stackrel{9}{\sigma}$ & 1 & बे & 2 \\
\hline $\begin{array}{l}\infty \\
11 \\
\infty\end{array}$ & $\stackrel{\Delta}{0}$ & 8 & 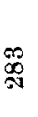 & $\stackrel{+}{\not}$ & $\stackrel{g}{g}$ & के & $\frac{\pi}{\infty}$ & $\begin{array}{c}0 \\
10 \\
6\end{array}$ & $\underset{\sigma}{\infty}$ & 1 & $\mathbb{\infty}_{\infty}^{+1}$ & 1 & $\stackrel{\infty}{\infty}$ & $\oiint_{n}^{\infty}$ \\
\hline$\underset{\mho}{\mathscr{Z}}$ & $\frac{\dot{0}}{8}$ & 1 & 1 & $i$ & $\underset{0}{\mathbb{N}}$ & 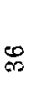 & {$[0$} & $\stackrel{0}{0}$ & 1 & $\infty^{\infty}$ & a & $E$ & & 1 \\
\hline$\|$ & 苂 & 8 & $\begin{array}{l}\infty \\
\stackrel{\infty}{-1}\end{array}$ & $\infty$ & is & $\infty$ & $\stackrel{\infty}{\infty}$ & $\stackrel{9}{0}$ & $\stackrel{s}{\circ}$ & $\sigma_{\infty}^{\infty}$ & تص & $0^{\infty}$ & & 1 \\
\hline $\mathbb{U}_{0}$ & $\stackrel{8}{8}$ & ] & $\mid$ & 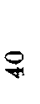 & م & $\stackrel{91}{\rightarrow}$ & $\frac{\infty}{\pi}$ & 1 & $\stackrel{\infty}{\infty}$ & $\cong$ & 1 & 1 & 1 & 1 \\
\hline${ }_{0}^{11}$ & 家 & 8 & $\underset{-1}{N}$ & $\underset{\infty}{\infty}$ & 2 & $\vec{\sigma}$ & 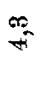 & $\stackrel{\infty}{-}$ & $\stackrel{10}{-10}$ & $\hat{0}$ & 1 & 1 & $\mid$ & 1 \\
\hline$\bigotimes_{\mathscr{W}}$ & مُ & 1 & 1 & $\stackrel{N}{n-1}$ & $\underset{\infty}{\infty}$ & 10 & 2 & 1 & 1 & $\infty_{0}$ & 1 & 1 & $\mid$ & 1 \\
\hline $\begin{array}{l}11 \\
\infty\end{array}$ & $\stackrel{B}{0}$ & 8 & $+\infty$ & $\infty$ & की & $\stackrel{+1}{-1}$ & $\sigma$ & 1 & 0 & 1 & 1 & 1 & 1 & 1 \\
\hline \multicolumn{2}{|c|}{ to } & 0 & $\ominus$ & $\stackrel{8}{8}$ & \& & 8 & 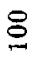 & 8 & 尺 & 号 & 8 & 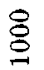 & $\underset{8}{8}$ & $\underset{\wp}{\varnothing}$ \\
\hline
\end{tabular}


Eine tabellarische Zusammenstellung der beobachteten und der berechneten Werte von $i$ gibt die Tabelle auf p. 761 .

Die beohachteten und die berechneten Werte stimmen für $\partial=60 \mathrm{sec}$ und $\delta=600 \mathrm{sec}$ gut überein (für $\delta=250000 \mathrm{sec}$ ist die ausgeglichene Kurve der beobachteten Werte als berechnete eingesetzt, daher die Übereinstimmung selbstverständlich); im übrigen ist zwar die GröBenordnung dieselbe, die Abweichung aber doch eine die Beobachtungsfehler übersteigende.

Dabei ist aber zu berücksichtigen, daB für kleine Ladungszeiten $(\delta=2 \mathrm{sec}$ und $10 \mathrm{sec})$ der eingesetzte Wert von $\delta$ ungenau ist, ferner daB in den verschiedenen Messungsreihen nicht dieselbe 'Temperatur zu erzielen war, was ebenfalls Voraussetzung der exakten Übereinstimmung ist.

Weitere Versuche prüfen die Richtigkeit der Annahme, daB bei verschiedenen Werten $E$ der elektromotorischen Kraft die zugehörigen Werte von $B$ in der Formel $i=B \cdot t^{-n}$ der elektromotorischen Kraft proportional sind.

Versuchsreihe 8.

Glirnmerkondensator von 1 Mikrof. Kapazität, Ladungsdauer $\delta=600 \mathrm{sec}, E$ variabel.

Die Versuchsergebnisse sind zusammengestellt in der folgenden Tabelle.

\begin{tabular}{|c|c|c|c|c|c|}
\hline \multirow{2}{*}{$\begin{array}{c}t \\
\text { (sec) }\end{array}$} & \multicolumn{5}{|c|}{$E=$} \\
\hline & 10 Volt & 20 Volt & 50 Volt & 100 Volt & 300 Volt \\
\hline 0 & - & - & - & - & - \\
\hline 30 & 4,8 & 8,5 & 22 & 47 & 一 \\
\hline 50 & 2,9 & 5,3 & 13,8 & 29 & 94 \\
\hline 60 & - & 4,3 & 11,6 & 24,1 & 77 \\
\hline 100 & 1,5 & 2,6 & 8,0 & 15,0 & 47 \\
\hline 250 & 0,53 & 1,09 & 2,83 & - & 19 \\
\hline 500 & 0,24 & 0,56 & 1,54 & 2,99 & 9,3 \\
\hline 1000 & 0,13 & 0,21 & 0,60 & 1,20 & 4,7 \\
\hline
\end{tabular}

Die Abweichungen liegen innerhalb der Grenzen der Beobachtungsfehler; die obige Annahme erweist sich also berechtigt bei Variationen der elektromotorischen Kraft im Verhältnis von $1: 30$. 
Anwendung der experimentell gefundenen Resultate.

Im folgenden soll an einem Beispiel gezeigt werden, wie aus der Untersuchung des Rückstandsstromes die dadurch bedingten Anomalien anderer Art numerisch berechnet werden können.

Aus Versuchsreihe 4 ergibt sich für einen Glimmerkondensator von 1 Mikrof. Kapazität bei $E=300$ Volt für den Rückstandsstrom:

$$
\begin{aligned}
i=B \cdot t^{-n} ; \quad B & =1370 \text { partes }=4,38 \cdot 10^{-7} A \\
n & =0,64 .
\end{aligned}
$$

Setzt man $B=C E \beta$, so erhält man:

$$
\beta=1,46 \cdot 10^{-3}\left(\frac{1}{\mathrm{sec}}\right)
$$

\begin{tabular}{|c|c|c|c|c|c|c|}
\hline$t=1$ & $\mathrm{sec}$ & 4,3 & $\mathrm{~m}$ & der & oni & idung \\
\hline 10 & $"$ & 9,5 & $"$ & $"$ & $"$ & $"$ \\
\hline 100 & $"$ & 20,9 & $"$ & $"$ & $"$ & , \\
\hline 1000 & $"$ & 46,0 & $n$ & $"$ & $"$ & $"$ \\
\hline
\end{tabular}

Die Rückstandsladung $R_{t}=\beta C E_{1-n}^{t^{1-n}}$ ist somit für

Für die Änderung der scheinbaren Kapazität $C^{\prime}$ mit der Periodendauer $\tau$ erhält man aus der Formel (vgl. p. 723)

$$
C^{\prime}=C\left[1+\left(\frac{\tau}{2} \bar{\pi}\right)^{1-n} \beta \Gamma(1-n) \cos \frac{(1-n) \pi}{2}\right]
$$

folgende Werte:

$$
\begin{aligned}
& \tau=0,0001 \mathrm{sec}, \quad \text { Frequenz }=10000, \quad C^{\prime}=1,000021 \mathrm{C} \\
& 0,001 \quad 1000 \quad 1,000046 \\
& 0,01 \quad 100 \quad 1,000106 \\
& 0,1 \quad 10 \quad 1,000243 \\
& \begin{array}{lll}
1,0 & 1 & 1,000556
\end{array}
\end{aligned}
$$

Analog ergibt sich aus der Formel für den reziproken scheinbaren Widerstand:

$$
L^{\prime}=L+\left(\frac{\tau}{2 \pi}\right)^{-n} \beta C \frac{\pi}{2 \Gamma(n) \cos \frac{(1-n)}{2} \pi}
$$


für den scheinbaren Widerstand des Kondensators infolge seiner Rückstandsbildung $\omega=1 /\left(L^{\prime}-L\right)$ für

$$
\begin{aligned}
& \tau=\begin{array}{cc}
0,0001 \mathrm{sec}, \\
0,001
\end{array} \quad \text { Frequenz }=\begin{array}{c}
10000, \\
1000
\end{array} \quad \omega=\begin{array}{l}
14000 \mathrm{Ohm} \\
61000
\end{array} \\
& \begin{array}{lll}
0,01 & 100 & 267000
\end{array} \\
& \begin{array}{lll}
0,1 & 10 & 1180000
\end{array} \\
& 1,0 \quad 1 \quad 5150000
\end{aligned}
$$

Diesem scheinbaren Widerstand entspricht dann die analog wie die Joulesche Wärme berechnete Energievergeudung im Kondensator.

Eine Zusammenstellung der Werte von $\beta$ und $n$, die in analoger Weise für andere Substanzen die auf Rückstandsbildung berubenden Phänomene numerisch berechnen läBt, ergibt:

Glimmer (Versuch 4) bei ca. $20^{\circ}$ C.:

$$
\beta=1,46.10^{-3}\left(\frac{1}{\mathrm{sec}}\right) ; \quad n=0,64 .
$$

Glas (Eprouvette, Versuch 3) bei ca. $20^{\circ} \mathrm{C}$. :

$$
\beta=78 \cdot 10^{-3}\left(\frac{1}{\sec }\right) ; \quad n=0,74 \text {. }
$$

Glas (Kochkolben, Versuch 6) bei ca. $18^{\circ} \mathrm{C}$ :

$$
\beta=15,4 \cdot 10^{-3}\left(\frac{1}{\mathrm{sec}}\right) ; \quad n=0,70 .
$$

Paraffinpapier (Versuch 5) bei ca. $20^{\circ}$ C.:

$$
\begin{gathered}
\beta=128 \cdot 10^{-3}\left(-\frac{1}{\text { sec }}\right) ; n=0,34 . \\
\text { Vierter Teil. } \\
\text { Zusammenfassung der Resultate. }
\end{gathered}
$$

I. Es wurde zunächst auf Grund der bisher vorliegenden experimentellen Ergebnisse eine Darstellung der Hauptformen anomalen Verhaltens der Dielektrika und dor dabei gefundenen GesetzmäBigkeiten gegeben. Als solche Hauptformen werden unterschieden:

1. die Rückstandsbildung;

2. die Energieverluste (Wärmeproduktion) in Dielektrikas unter dem Einflub eines Wechselfeldes;

3. die ponderomotorischen Kräfte, die ein Dielektrikum in einem relativ zu ihm rotierenden Felde erfährt; 
4. die scheinbare Abhängigkeit der Kapazität eines Kondensators von Ladangsdauer bei konstanter Spannung, bzw. Periodendauer bei Wechselspannung.

II. Es wurde hierauf gezeigt, daß das Vorhandensein von Rückstandsbildung in einem Medium notwendig auch die unter 2. bis 4. genannten Formen anomalen Verbaltens bedingt und daB aus den empirisch gefundenen Gesetzen der Rückstandsbildung auch die Gesetze für diese Formen quantitativ ableitbar sind.

Es wird ferner gezeigt, daß zwar ohne Rückstandsbildung diesen anderen Formen analoge Erscheinungen durch Leitung des Dielektrikums zustande kommen können, daß aber eine Berücksicbtigung der bisher gefundenen Ergebnisse quantitativen Charakters diese Erklärung als unzureichend erscheinen läBt.

Die Diskussion der verschiedenen Möglichkeiten, die Anomalien der Dielektrika theoretisch zu behandeln, führt auf folgende Resultate:

Die Annahme inhomogener Struktur des Dielektrikums (Maxwellsche Theorie der geschichteten Dielektrika) führt zu qualitativ richtigen Folgerungen, doch ist infolge mathematischer Schwierigkeiten eine exakte Lösung der gestellten Probleme und damit ein Vergleich von Theorie und Erfahrung in quantitativer Beziehung vorläufig undurchführbar.

Die Annahme anomaler Leitung (Ionenleitung) führt teilweise zu qualitativ richtigen Ergebnissen, teilweise aber zu Konsequenzen, die mit der Erfahrung im Widerspruch stehen. Anomale Leitung kann daher in manchen Fällen an den beobachteten Erscheinungen mitbeteiligt sein, obne eine ausreichende Erklärung zu liefern.

Die Annahme anomaler Vorgänge dielektrischer Natur (dielektrische Nachwirkung, Hysteresis) ist unzureichend in der Form, wie sie bei den Erscheinungen der magnetischen Hysteresis mit Erfolg verwendet wurde. Die von Pellat präzisierte Annahme einer dielektrischen Nachwirkung (viskosen Hysteresis), daB die dielektrische Verschiebung nach einem bestimmten einfachen Gesetz ihres zeitlichen Verlaufes einem der jeweiligen Feldintensität proportionalen stationären Endwert zustrebe

$$
\left(\frac{d \mathscr{D}}{d t}=K^{\frac{d \mathfrak{E}}{d t}}-\frac{d}{d t}[\mathcal{D}-\varepsilon K \mathfrak{E}]\right)
$$


gibt im allgemeinen eine gute Annäherung an die beobachteten Erscheinungen, doch bleiben in einzelnen Punkten Differenzen zwischen Theorie und Beobachtung bestehen.

Es wird nun eine Modifikation dieser Theorie durchgeführt, die darin besteht, daB die dielektrische Verschiebung in eine Summe beliebig vieler Glieder zerlegt wird, von denen jedes einzelne demselben einfachen Gesetz folgt wie nach der Pellatschen Theorie, doch unter Variation der in der Formel enthaltenen Parameter.

Es folgt eine Interpretation der dieser Theorie zugrunde liegenden Annahmen rom molekularphysikalischen Standpunkte aus.

III, Experimentelle Untersuchungen der Rückstandsbildung mittels einer galvanometrischen Methode bestätigen und ergänzen die schon von anderen Autoren gefundenen Resultate bezüglich des zeitlichen Ganges der Rückstandsbildung, der Gültigkeit des Superpositionsprinzips, des Einflusses der Temperatur und der Proportionalität zwischen Rückstandsstrom und Spannung.

An einem Beispiel wird gezeigt, wie aus zwei Konstanten, deren Werte aus der Untersuchung des Rückstandsstromes ermittelt wurden, die anderen Formen anomalen Verhaltens durch Angabe numerischer Werte dargestellt werden können.

\section{Literatur.}

I. Rückstand.

1. R. Kohlrausch, Pogg. Ann. 91. p. 56. 1854.

2. W. v. Bezold, Pogg. Ann. 114. p. 437. 1861.

3. A. Gaugain, Ann. chim. phys. (4) 2. p. 276, 313. 1864.

4. R. Clausius, Abh. über meeh. Wärmeth. Il. p. 135-162. 1867.

5. J. C. Maxwell, Treatise I. p. 374. 1873.

6. A. Wüllner, Pogg. Ann. 153. p. 22. 1874.

7. L. Boltzmann, Romich u. Nowack, Wiener Sitzungsber. 70. p. 381.1874 .

8. B. Riemann, Ges. Werke p. 48, 345, 353. 1876.

9. J. Hopkinson, Phil. Trans. 166. p. 489. 1876.

10. - Phil. Mag. (5) 2. p. 314. 1876; auch Proc. Roy. Soc. 25. p. 496. $1876 / 77$.

11. - Phil. Trans. 167. p. 599. 1877/78.

12. A. Wüllner, Wied. Ann. 1. p. 247. 1877. 
13. H. A. Rowland, Americ. J. of Math. 1. p. 53. 1878.

14. W. E. Ayrton u. J. Perry, Proc. Roy. Soc. 27. p. 238.1878.

15. W. Giese, Wied. Ann. 9. p. 161. 1880.

16. H. A. Rowland u. E. L. Nichols, Phil. Mag. (5) 11. p. 414. 1881.

17. H. Hertz, Wied. Ann. 20. p. 279. 1883.

18. C. Dieterici, Wied. Ann. 25. p. 545. 1885.

19. A. Wüllner, Wied. Ann. 32. p. 19. 1887.

20. L. Arons, Wied. Ann. 35. p. 291. 1888.

21. J. Curie, Ann. chim. phys. (6) 18. p. 203. 1889.

22. H. Muraoka, Wied. Ann. 40. p. 328. 1890.

23. E. Bouty, Compt. rend. 110. p. 846, 1362. 1890; auch Ann. chim. phys. (6) 27. p. 62.1892.

24. A. Hess, Lum. electr. 46. p. 401, 507. 1892; auch Journ. de Phys. (3) 2. p. 145. 1893.

25. B. Dessau, Rend. R. Acc. d. Linc. (5) 2. II. p. 86. 1893.

26. F. Bedell u. C. Kinsley, Phys. Rev. 2. p. 170. 1894.

27. R. Lombardi, Mem. R. Acc. Torino (2) 44. 1894.

28. A. S. Dunstan, Electr. World 26. p. 3. 1895.

29. A. Hess, Eclair. electr. 4. p. 205. 1895.

30. - Eclair electr. 7. p. 450. 1896.

31. H. V. Carpenter, Phys. Rev. 4. p. 238. 1896.

32. Th. Wulf, Wiener Sitzungsber. 105. p. 667. 1896.

33. L. Houllevigue, Journ. de Phys. (3) 6. p. 113, 120, 153. 1897.

34. J. Hopkinson u. E. Wilson, Proc. Roy. Soc. 60. p. 425.1897.

35. F. Hasenöhrl, Wiener Sitzungsber. 107. p. 1035. 1898.

36. H. A. Rowland u. T. D. Penniman, J. Hopk. Un. Circ. 17. p. 52. 1898.

37. A. Naceari, Atti Torino 34. p. 1088. 1899.

38. L. M. Potts, J. Hopk. Un. Circ. 18. p. 59. 1899.

39. H. Pellat, Compt. rend. 128. p. 1312. 1899; auch Ann. ehim. phys. (7) 18. p. 150. 1899.

40. L. M. Potts, J. Hopk. Un. Circ. 19. p. 62.1900.

41. H. Pellat, Journ. de Phys. (3) 9. p. 313. 1900.

42. C. V. Drysdale, Electrician 46. p. 890. 1901.

43. A. W. Ashton, Phil. Mag. (6) 2. p. 501. 1901.

44. J. Buchanan, Phil. Mag. (6) 3. p. 240, 1902.

45. E. v. Schweidler, Wiener Sitzungsber. 111. p. 573. 1902.

46. -1. c. p. 579 .

47. U. Seiler, Mitt. Phys. Ges. Zürich Nr. 3. p. 12. 1902.

\section{Hysteresis.}

1. W. Siemens, Pogg. Ann. 125. p. 137. 1864.

2. A. Naccari u. F. Bellati, Journ. de Phys. (2) 1. p. 430. 1882.

3. J. Borgmann, Journ. d. russ. phys.chem. Ges. (1) 18. p.6. 1886; auch Journ. de Phys. (2) 8. p. 217. 1888.

4. J. Trowbridge u. W. C. Sabine, Phil. Mag. (5) 30. p. 323. 1890; auch Phys. Rev. 1. p. 183. 1892. 
5. J.Trowbridge u. W. C. Sabine, Rep. Brit. Ass. Leeds p. 781.1890.

6. C. P. Steinmetz, Electrician 28. p. 384, 408, 425. 602. 1892; auch Lum. electr. 44. p. 95. 1892 ; auch Elektrotechn. Zeitschr. 13. p. 227. 1892.

7. R. Arno, Rend. R. Acc. dei Linc. 1. p. 284.1892.

8. P. Janet, Journ. de Phys. (3) 2. p. 337, 1893.

9. A. Hess, Lum. electr. 47. p. 466. 1893; auch Journ. de Phys. (3) 2. p. 145.1893.

10. G. Benischke, Wiener Sitzungsber. 102. p. 1345. 1893.

11. A. Kleiner, Wied. Ann. 50. p. 138. 1893.

12. C. Borel, Compt. rend. 116. p. 1192. 1893; auch Arch. de Genève (3) 30. p. 45.1893.

13. W. Weiler, Zeitschr. f. phys. u. chem. Unt. 7. p. 1. 1893.

14. H. Fritz, Diss. Zürich 1893.

15. R. Arno, Cim. (3) 33. p. 15. 1893.

16. - Rend. R. Acc. dei Linc. (5) 2. I. p. 341. 1893.

17. - 1. c. (5) 2. II. p. 260.189 .

18. - J. c. (5) 3. I. p. 585.1894.

19. - l. e. (5) 3. II. p. 294. 1894.

20. A. Kleiner, Arch. de Genève (3) 32. p. 282.1894.

21. H. Düggelin, Vierteljahrsschr. d. naturf. Ges. Zürich 40. p. 121. 1895.

22. A. W. Porter u. D. K. Morris, Proc. Roy. Soc. 57. p. 469.1895.

23. A. Hess, Eclair. electr. 4. p. 205.1895.

24. G. Benischke, Zeitschr. f. Elektrotechn. Heft 16. 1895.

25. H. Eisler, Zeitschr. f. Elektroteehn. Heft 16. 1895.

26. H. F. Weber, Arch. de Genève (4) 2. p. 519, 1896.

27. R. Arno, Rend. R. Acc. de Linc. (5) 5. I. p. 262.1896 ; auch N. Cim. (4) 5. p. 52.1896.

28. A. Hess, Eclair. electr. 7. p. 450. 1896.

29. L. Houllevigue, Journ. de Phys. (3) 6. p. 120.1897.

30. R. Threlfall, Phys. Rev. 4. p. 457. 1897.

31. - l. e. 5. p. $21,65.1897$.

32. W. Schaufelberger, Diss. Zürich 1898; auch Wied. Ann. 65. p. 635.1898.

33. H. Pellat, Compt. rend. 128, p. 1312. 1899; auch Ann. chim. phys. (7) 18. p. 150. 1899.

34. W. Schaufelberger, Wied. Ann 67. p. 307. 1899.

35. E. B. Rosa u. A. W. Smith, Phys. Rev. 8. p. 1. 1899; auch Phil. Mag. (4) 47. p. 222.1899.

36. F. Beaulard, Compt. rend. 130. p. 1182.1900.

37. - Journ. de Phys. (3) 9. p. 422. 1900.

38. H. Pellat u. F. Beaulard, Compt. reod. 130. p. 1457. 1900.

39. - Journ. de Phys. (3) 9. p. 313. 1900.

40. L. M. Potts, J. Hopk. Un. Circ. 19. p. 62. 1900; auch Phys. Zeitschr. 2. p. 301. 1900.

41. P. L. Mercanton, Bull. soc. vaud. Oktober 1901; auch Journ. de Phys. (4) 1. p. 33. 1902. 
42. C. P. Steinmetz, Electric. World 37. p. 1065. 1901; auch Eclair. electr. 29. p. 27. 1901; Electrician 47. p. 412. 1901; Nat. Rundsch. 16. p. 597. 1901.

43. W. E. Ayrton, Electrician 46. p. 476. 1901.

44. A. W. Ashton, Phil. Mag. (6) 2. p. 501. 1901.

45. F. Macearone, N. Cim. (5) 2. p. 88. 1901; auch Physik. Zeitschr. 3. p. 57. 1901.

46. C. E. Skinner, Electr. Rev. 41. p. 82. 1902.

47. F. Maccarone, N. Cim. (5) 4. p. 313. 1902.

48. F. Beaulard, Congrès d'Angers de l'assoc. franç. pour l'avance des sciences 1903; auch Eclair. electr. 37. p. 404. 1903.

49. J. Moscicki u. M. Altenberg, Krak. Anz. p. 46. 1904.

50. C. E Guye u. P. Denso, Arch, de Genève 19. p. 101. 1905; auch Compt. rend. 140. p. 433. 1905.

51. O. M. Corbino, N. Cim. (5) 9. p. 81. 1905; auch Physik. Zeitschr. 6. p. 138. 1905 .

52. V. v. Lang, Wiener Sitzungsber. 115. p. 211. 1906.

\section{Rotation und Drehmomente.}

1. H. Hertz, Wied. Ann. 13. p. 266. 1881.

2. G. Quincke, Wied. Ann. 59. p. 417. 1896.

3. A. Heydweiller, Verh. d. Pbysik. Ges. Berlin 16. p. 32.1897.

4. A. Boltzmann, Wied. Ann. 60. p. 399. 1897.

5. E. v: Schweidler, Wiener Sitzungsber. 106. p. 526. 1897.

6. A. Heydwoiller, Wied. Anv. 69. p. 531. 1899.

7. L. Graetz, Ann. d. Phys 1. p. 530. 1900.

Vgl. auch Arno, II, 7, 15-19, 27; Weiler, II, 13; Threlfall, II, 30, 31; Schaufelberger, II, 32, 34; v. Lang, II, 52.

\section{Anomale Ireitung.}

1. H. Hertz, Wied. Ann. 20. p. 279. 1883.

2. G. Quincke, Wied. Ann. 28. p. 529. 1886.

3. H. Koller, Wiener Sitzungsber. 98. p. 201. 1889.

4. R. Appleyard, Phil. Mag. (5) 38. p. 396. 1894.

5. - Phil. Mag. (5) 42. p. 148. 1896.

6. A. Naccari, N. Cim. (4) 8. p. 259.1898.

7. E. V. Schweidler, Wiener Sitzungsber. 109. p. 964.1900.

8. - Ann. d. ihys. 5. p. 483. 1901.

9. H. Gädeke, Diss. Heidelberg 1901.

10. E. v. Schweidler, Wiener Sitzungaber. 113. p. 881. 1904.

11. R. Appleyard, Phil. Mag. (6) 10. p. 485.1905.

12. P. Gouré de Villemontée, Compt. rend. 141. p. 179. 1905.

\section{Abhängigkeit der Dielektrizitätskonstante von Ladungs- dauer oder Wechselzahl.}

1. N. Schiller, Pogg. Ann. 152. p. 555. 1872.

2. L. Boltzmann, Wiener Sitzungsber. 67. p. 17. 1873. 
3. L. Boltzmann, Wiener Sitzungsber. 68. p. 81. 1873.

4. - Wiener Sitzungsber. 70. p. 307. 1874.

5. J. J. Thomson, Proc. Roy. Soc. 46. p. 292.1889.

6. E. Lecher, Wiener Sitzungsber. 99. p. 480. 1890; auch Wied. Ann. 42. p. 142. 1891 .

7. R. Blondlot, Compt. rend. 112. p. 1058. 1891.

8. L. Arons u. H. Rubens, Wied. Ann. 42. p. 581.1891.

9. - - Wied. 44. p. 206. 1891.

10. A. Perot, Compt. rend. 115. p. 38, 165. 1892.

11. J. Sahulka, Wiener Sitzungsber. 102. p. 773.1893.

12. D. Mazotto, Rend. R. Acc. dei Linc. (5) 4, I. p. 240.1893.

13. B. W. Stankewitsch, Wied. Ann. 52. p. 700. 1894.

14. L. Graetz u. L. Fomm, Münchener Ber, 24. p. 184. 1894; anch Wied. Ann. 54, p. 626. 1895.

15. F. Beaulard, Compt. Rend. 119. p. 268. 1894.

16. C. Heinke, Wied. Ann. 54. p. 577. 1895.

17. E. Northrup, Phil. Mag. (5) 39. p. 78.1895.

18. Di Mazotto, N. Cim. (4) 1. p. 308, 1895.

19. A. Lampa, Wiener Sitzungsber. 105. p. 587, 1049. 1896; auch Wied. Ann. 61. p. 79. 1897.

20. E. S. Ferry, Phil. Mag. (5) 44. p. 404. 1897.

21. P. Drude, Zeitschr. f. physik. Chem. 23. p. 267. 1897.

22. J. Hanauer, Wied. Ann. 65. p. 577. 1898.

23. F. Marx, Wied. Ann. 66. p. 411. 1898.

24. W. C. Hormell, Phil. Mag. (6) 3. p. 52. 1902.

25. J. Kosogonoff, Physik. Zeitsehr. 3. p. 207. 1902.

26. A. de Forest Palmer, Phys. Rev. 16. p. 267. 1903.

27. H. C. Parker, Ann. New York Ac. 15. p. 161. 1904.

\section{Varia.}

1. E. Root, Pogg. Ann. 158. p. 1, 425. 1876.

2. M. v. Hoor, Elektrotechn. Zeitschr. 22. p. 170, 187, 213, 716, 749, 781. 1901.

3. K. Germanischskaja, Diss. Zürich 1903.

4. V. Cremieu u. L. Malcles, Compt. rend. 139. p. 790. 1904.

(Eingegangen 15. Oktober 1907. 